\title{
Hypoxia-induced p53 modulates both apoptosis and radiosensitivity via $\mathrm{AKT}$
}

\author{
Katarzyna B. Leszczynska, ${ }^{1}$ losifina P. Foskolou, ${ }^{1}$ Aswin G. Abraham, ${ }^{1}$ Selvakumar Anbalagan, ${ }^{1}$ Céline Tellier, ${ }^{1}$ Syed Haider, \\ Paul N. Span, ${ }^{2}$ Eric E. O'Neill, ${ }^{1}$ Francesca M. Buffa, ${ }^{1}$ and Ester M. Hammond' \\ 'Cancer Research UK and Medical Research Council Oxford Institute for Radiation Oncology, Department of Oncology, University of Oxford, Oxford, United Kingdom. ²Radboud University Medical Centre, \\ Department of Radiation Oncology 874, Nijmegen, Netherlands.
}

\begin{abstract}
Restoration of hypoxia-induced apoptosis in tumors harboring $\mathrm{p} 53$ mutations has been proposed as a potential therapeutic strategy; however, the transcriptional targets that mediate hypoxia-induced $\mathrm{p} 53$-dependent apoptosis remain elusive. Here, we demonstrated that hypoxia-induced p53-dependent apoptosis is reliant on the DNA-binding and transactivation domains of $\mathrm{p} 53$ but not on the acetylation sites $\mathrm{K} 120$ and $\mathrm{K} 164$, which, in contrast, are essential for DNA damage-induced, p53-dependent apoptosis. Evaluation of hypoxia-induced transcripts in multiple cell lines identified a group of genes that are hypoxia-inducible proapoptotic targets of $\mathrm{p} 53$, including inositol polyphosphate-5-phosphatase (INPP5D), pleckstrin domaincontaining A3 (PHLDA3), sulfatase 2 (SULF2), B cell translocation gene 2 (BTC2), cytoplasmic FMR1-interacting protein 2 (CYFIP2), and KN motif and ankyrin repeat domains 3 (KANK3). These targets were also regulated by $\mathrm{p} 53$ in human cancers, including breast, brain, colorectal, kidney, bladder, and melanoma cancers. Downregulation of these hypoxia-inducible targets associated with poor prognosis, suggesting that hypoxia-induced apoptosis contributes to $\mathrm{p} 53$-mediated tumor suppression and treatment response. Induction of $\mathrm{p} 53$ targets, PHLDA3, and a specific INPP5D transcript mediated apoptosis in response to hypoxia through AKT inhibition. Moreover, pharmacological inhibition of AKT led to apoptosis in the hypoxic regions of p53-deficient tumors and consequently increased radiosensitivity. Together, these results identify mediators of hypoxiainduced p53-dependent apoptosis and suggest AKT inhibition may improve radiotherapy response in p53-deficient tumors.
\end{abstract}

\section{Introduction}

Regions of low/insufficient oxygen (hypoxia) are a pathophysiological feature of most solid tumors (1). It has been demonstrated conclusively that patients with significant levels of tumor hypoxia have a worse prognosis irrespective of treatment modality (2-4). Within a tumor, gradients of oxygen concentrations exist, which are likely to reach complete anoxia in avascular areas $(5,6)$. Cells exposed to severe hypoxia $\left(<0.1 \% \mathrm{O}_{2}\right)$ are particularly radioresistant and induce a replication stress-mediated DNA damage response (DDR), which includes stabilization and activation of the p53 tumor suppressor (7). Replication stress refers to the slowing or stalling of replication forks (8). Importantly, hypoxia induces a DDR in the absence of detectable DNA damage (9). p53 is rapidly stabilized and activated in response to a wide variety of cellular stresses, including DNA damage, oncogene expression, nutrient deprivation, ribosomal dysfunction, and hypoxia $\left(<0.1 \% \mathrm{O}_{2}\right)(10)$. Tumor hypoxia is potentially one of the earliest driving forces to mutate p53 function during tumorigenesis (11). The majority of human cancers (over 50\%) have lost WT p53 function due to mutations in the DNA-binding domain (DBD) of the protein (10, 12). We recently demonstrated that even mild levels of hypoxia $\left(2 \% \mathrm{O}_{2}\right)$ combined with oncogene-induced replication stress can

\section{Related Commentary: p. 2264}

Conflict of interest: The authors have declared that no conflict of interest exists. Submitted: December 8, 2014; Accepted: March 19, 2015.

Reference information: J Clin Invest. 2015;125(6):2385-2398. doi:10.1172/JCI80402. lead to DDR signaling and that this can occur before the accumulation of DNA damage (7).

Under conditions of hypoxia-induced replication stress, p53 is phosphorylated and stabilized by the ATR and, to a lesser extent, ATM kinases $(9,13)$. In addition, p53 stabilization is facilitated by decreased expression of MDM2 and/or by phosphorylation of MDMX E3 ubiquitin ligases $(14,15)$. Once activated by hypoxia, p53 induces apoptosis through the intrinsic pathway $(16,17)$ and requires the transcriptional activity of p53 $(18,19)$. However, the exact p53 target genes responsible for apoptosis in hypoxia remain poorly characterized. Isolated reports exist, which describe the involvement of known proapoptotic targets, such as PUMA or BAD, in hypoxiainduced and p53-dependent apoptosis, although these tend to be restricted to specific cell lines/types, for example, cardiomyocytes (20). In contrast, a number of reports demonstrate that genes such as PUMA, BAX, or DR5 are not induced by $\mathrm{p} 53$ in various cancer cell lines during hypoxia-induced apoptosis $(18,21,22)$. Previous studies have suggested that p53 acts predominantly as a transrepressor rather than a transactivator during hypoxia (18-21). However, few targets of p53-mediated transrepression have been reported (18-21). Interestingly, recent studies investigating the tumor-suppressive function of p53 demonstrated that the most characterized p53 targets crucial for DNA damage-induced cell cycle arrest, senescence, or apoptosis ( $p 21$, PUMA, NOXA) appear to be dispensable for suppressing tumorigenesis (23-25). Instead, p53 targets involved in regulation of energy metabolism, ROS production, and autophagy 
(e.g., TIGAR, GLS2, or ULK1) appear to be crucial in tumor suppression (24-26). Recently, using genetically engineered mouse knockin models in the transactivation domains of p53, Brady et al. demonstrated that the genes critical for the acute DDR of 553 and for tumor suppression differ. Brady et al. identified 14, mostly novel, p53 targets required for p53-mediated tumor suppression (23). The induction of these genes was found to be dependent on both of the transactivation domains of p53 and an intact DBD. More recently, additional mouse models showed that acetylation of p53 at specific lysine residues K117/K161/ K162 (corresponding to K120/K164 in humans) in the DBD are essential for DNA damage-induced apoptosis but are dispensable for p53-dependent tumor suppression (24). These studies have led to the conclusion that p53-induced apoptosis is not required for tumor suppression.

In this study, we investigated the p53-mediated transcriptional response to hypoxia and specifically the mechanism of p53-induced apoptosis in these conditions. Hypoxia-induced p53-dependent apoptosis was reliant on DNA binding (R175) but not acetylation in the DBD (K120, K164). We show that a group of 6 validated hypoxia-inducible p53 target genes was found to predict $\mathrm{p} 53$ status in samples from patients with cancer; in addition, we show that when these genes were underexpressed they correlated with poor clinical outcome in two independent analyses of large human breast cancer cohorts, with a total of more than 3,000 patients, and other human cancers. Two of the p53 targets induced in hypoxia, pleckstrin domain-containing A3 (PHLDA3) and inositol polyphosphate-5-phosphatase (INPP5D, which encodes SHIP-1), were found to induce apoptosis through inhibition of AKT signaling. We show that by mimicking the function of WT p53 in hypoxia using a pharmacological inhibitor of AKT we can increase apoptosis specifically in hypoxic areas of tumors with nonfunctional p53 but not in tumors with WT p53. Furthermore, increased apoptosis in the hypoxic fraction induced through AKT inhibition correlated with significant radiosensitization in an esophageal tumor model.

\section{Results}

Hypoxia-induced p53 transactivates specific target genes. Using hypoxia-inducible p53 constructs in p53-null H1299 cells, we investigated the role of DNA binding and acetylation of p53 in hypoxia-induced apoptosis (18). Mutation of the acetylation sites in the p53 DBD (K120R, K164R, K120R/K164R) had no significant effect on the level of hypoxia-induced apoptosis, in contrast to a structural mutation of the DBD (R175H), which prevented DNA binding and abrogated apoptosis entirely (Figure 1, A and B). However, in agreement with previous reports, acetylation at K120 and/or K164 was critical for p53-dependent apoptosis in response to camptothecin-induced DNA damage (Supplemental Figure 1, A and B) (27). These data immediately suggested that the mechanism of hypoxia-induced p53dependent apoptosis differs from the response to DNA-damaging agents $(24,27)$. Using the same inducible system, we carried out an expression array to compare the transcriptional response to WT p53 and p53 $3^{\mathrm{R} 175 \mathrm{H}}$ in hypoxia. As expected, the wellcharacterized p53 proapoptotic targets, including BAX, PUMA, NOXA, or $B I D$, were not upregulated by 553 in response to hypoxia (18). Instead, a number of other p53 targets were identified, of which the most significant are shown in Figure 1C (http://www.ncbi.nlm.nih.gov/geo/; accession number GSE53893). Notably and in contrast to our previous study in myc/rastransformed mouse embryonic fibroblasts, we did not observe significant levels of p53-mediated gene repression (18). PHLDA3, one of the most significantly induced genes in hypoxia, was previously identified as 1 of 14 genes critical for p53-mediated tumor suppression (23). This led us to ask whether any of the other p53 targets identified by Brady et al. were also hypoxia regulated. PHLDA3 stood out as the most hypoxia inducible from this group of 14 genes; however, $\mathrm{KN}$ motif and ankyrin repeat domains 3 (KANK3), ABHD4, and DEF6 were also potential p53 targets in hypoxia, with some cell line dependence (Figure 1D and Supplemental Figure 1, C-E). GLUT1 served as hypoxia-inducible control and $B A X$ as a typical DNA damage-inducible p53 target. Together, these data support our hypothesis that p53 induces a stress-specific transcriptional response in hypoxia. Most importantly, these data suggest that p53-dependent apoptosis in response to hypoxia could still play a role in tumor suppression.

We focused on genes that have been linked with apoptosis (INPP5D,PHLDA3, sulfatase2 [SULF2], B celltranslocationgene2 [BTG2], cytoplasmic FMR1-interacting protein 2 [CYFIP2]) or tumor suppression (KANK3) $(23,28-31)$. Using quantitative PCR (qPCR), we validated these genes as being hypoxia inducible in a p53-dependent manner in the HCT116 isogenic cell lines $\left(p 53^{+/+}\right.$and $\left.p 53^{-/-}\right)$(Figure $\left.2 \mathrm{~A}\right)$. We also demonstrated that, in addition to the DBD, the transactivation domains of $\mathrm{p} 53$ ( $553^{22}, \mathrm{p} 53^{23}, \mathrm{p} 53^{53}, \mathrm{p} 53^{54}$ ) were required to induce these targets in hypoxia (Supplemental Figure 2, A and B). p53 response elements (REs) have been identified in PHLDA3, SULF2, CYFIP2, and $K A N K 3$, so we used ChIP to determine whether p53 was enriched at these sites in hypoxia (Figure 2B and refs. 32-34). In each case, there was a clear accumulation of p53 at the REs in hypoxia, demonstrating direct transactivation of these targets in response to hypoxia as well as the DNA-damaging stresses previously reported. In addition, we confirmed that PHLDA3 and SULF2 proteins were induced in hypoxia in a p53-dependent manner, again using $p 53^{+/+}$and $p 53^{-/-}$HCT116 cells or RKO cells treated with siRNA to p53 (Supplemental Figure 2, C-E). We also observed a hypoxic induction of both PHLDA3 mRNA and PHLDA3 protein in a human nontumor lung fibroblast cell line, WI38, suggesting that this regulation is not restricted to tumor cells (Supplemental Figure 2, F and G).

Previous reports suggest an interaction between p53 and HIF-1 $\alpha$ (35). We have determined that PHLDA3 and INPP5D were induced in response to $<0.1 \% \mathrm{O}_{2}$ when both p53 and HIF- 1 were induced but not in response to $2 \% \mathrm{O}_{2}$ when only HIF-1 was stabilized (Supplemental Figure 3, A-C). This was further verified in $H I F 1 A^{+/+}$and $H I F 1 A^{-/}$RKO cells, in which PHLDA3 and INPP5D were highly induced by hypoxia irrespective of HIF-1 $\alpha$ status, as opposed to the HIF-1 $\alpha$ target, GLUT1, which was not induced in the $\mathrm{HIF} \mathrm{A}^{-/-}$cell line (Supplemental Figure 3, D-G). This demonstrates that HIF-1 $\alpha$ does not contribute to the induction of the hypoxia-induced p53-dependent genes described.

Hypoxia-induced p53 specifically transactivates the INPP5D transcript associated with stem cells. INPP5D has been recently 
A

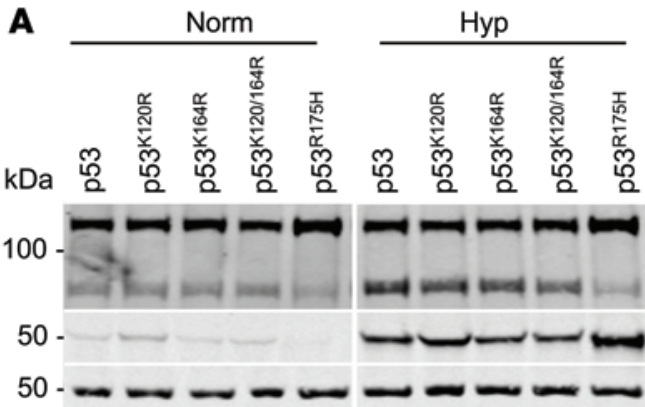

C
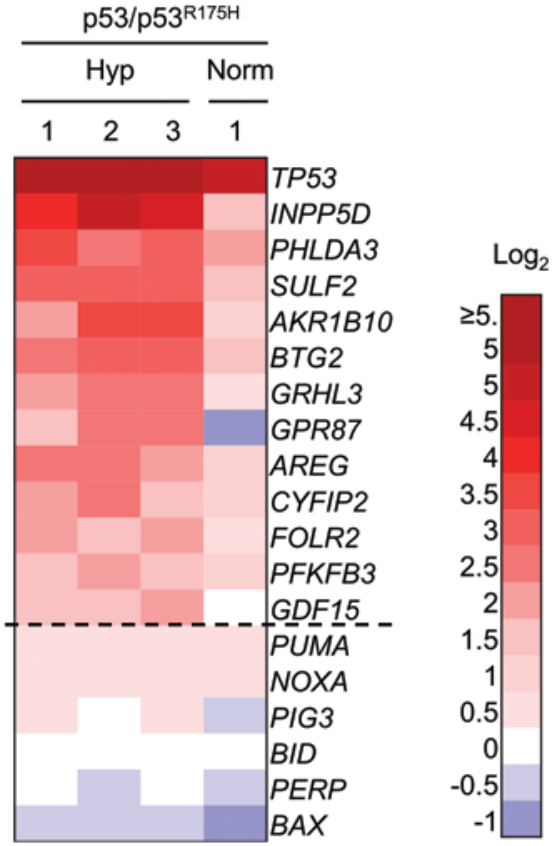

PARP

p53

$\beta$-Actin
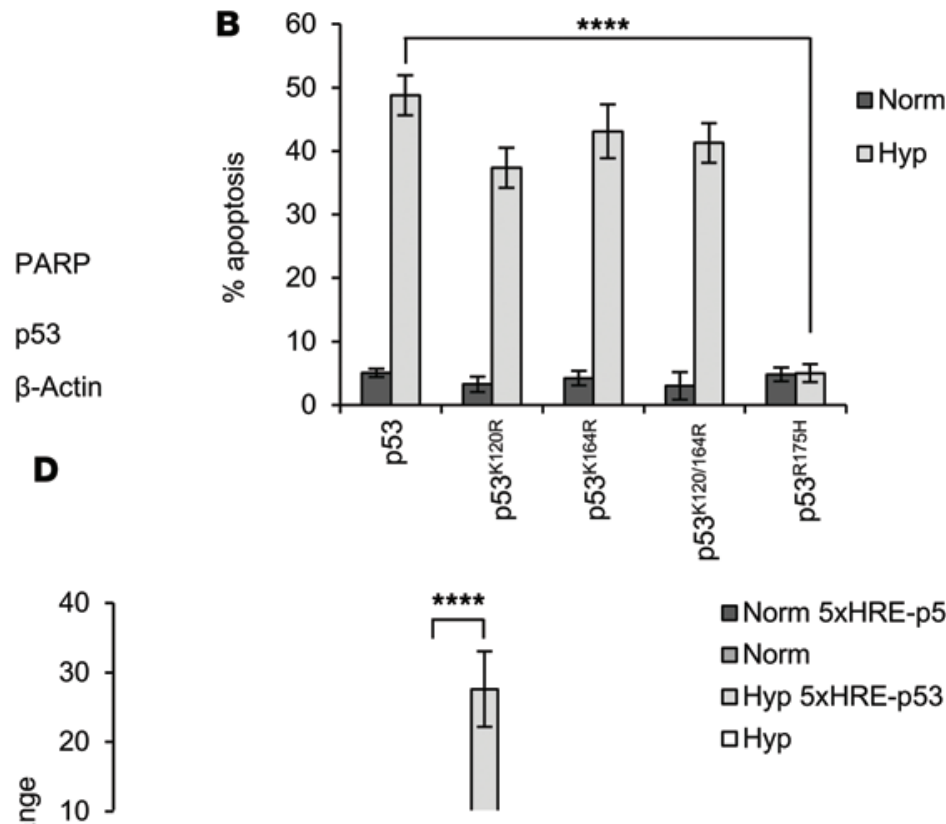

口Norm 5xHRE-p53 aNorm 口Hyp 5xHRE-p53 口Hyp

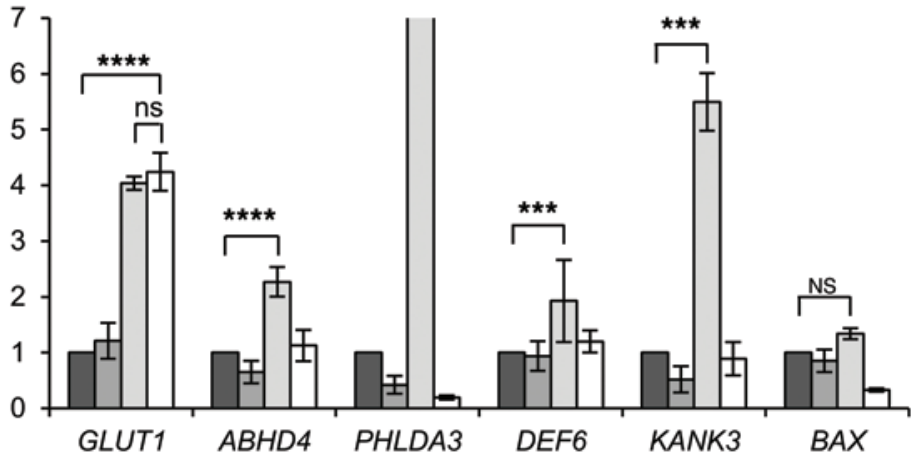

Figure 1. Hypoxia-induced p53-dependent apoptosis is associated with the expression of specific genes. (A and B) Apoptosis in H1299 cells expressing either WT p53 (p53) or the mutants (K120R, K164R, K120/164R, R175H) from the 5xHRE promoter and exposed to 24 hours of hypoxia (Hyp) or normoxia (Norm) detected by PARP cleavage or morphologically, respectively. For Western blotting, the lanes were run on the same gel but were noncontiguous. The bar graph shows mean \pm SEM (combined $n=3$, 2-way ANOVA test [ $\left.{ }^{* * *} P<0.0001\right]$ ). (C) Microarray gene expression analysis in H1299 cells transfected with either 5xHRE-p53 or 5xHRE-p53 $3^{\mathrm{R} 15 \mathrm{H}}$ and exposed to 16 hours of hypoxia. Heat map showing the top candidate genes induced by hypoxia in a p53dependent manner. Well-characterized proapoptotic p53 targets are shown in the bottom 6 rows. Fold change ratios of gene expression for $5 x H R E-$ $p 53 / 5 \times H R E-p 53^{R 175 H}$ samples are shown in red for upregulated and blue for downregulated genes. Columns numbered 1 to 3 represent technical replicates of hypoxic samples. (D) qPCR testing of the hypoxic regulation of p53 target genes identified as being essential to its tumor-suppressive role by Brady et al. (22) in H1299 cells transfected with 5xHRE-p53 and exposed to 24 hours of hypoxia. The bar graph shows mean \pm SEM (combined $n=3,2$-way ANOVA test $[P<0.0001]$ followed by 2 -tailed Student's $t$ test $\left[{ }^{* *} P<0.001 ;{ }^{* * *} P<0.0001\right]$.

described as a p53 target gene with 2 p53 REs in intron 9 (36). We carried out ChIP analysis using both these REs and found that one of them was moderately enriched for p53 binding in hypoxia (Supplemental Figure 4A). To investigate further and as multiple INPP5D transcript variants exist, we asked which transcript was induced in hypoxia (37). By carrying out PCR with specific primers, we determined that RKO and HCT116 cells expressed only the short INPP5D transcript (U50040), which was further induced by hypoxia (Figure $2 \mathrm{C}$ and Supplemental Figure $4 \mathrm{~B})$. This particular transcript is transcribed from an internal promoter in intron 5 of the full-length INPP5D gene (37). We aligned this region with the corresponding mouse and rat loci of the INPP5D gene and found a region of conserved similarity in the sequence directly preceding the cDNA of the short INPP5D. Within this region, we identified a novel putative p53 RE, which was conserved between species (chr2:233,139,693-233,139,712 of hg38 human genome assembly) (Figure 2D). Using ChIP, we verified that this sequence is a bona fide p53 RE and that occupancy of this region by p53 was enhanced in hypoxia to a similar level as that seen for PHLDA3, SULF2, CYFIP2, or KANK3 (Figure $2 \mathrm{E}$ ). The closely related member of the INPP5D gene family, INPPL1, did not increase in hypoxia (Supplemental Figure 4C). The expression of short INPP5D has been previously characterized mainly in mouse embryonic stem cells, although the mechanism of induction is unclear (37). We found that expression of this particular transcript was increased in response to hypoxia, not only in cancer cells but also in embryonic stem cells (Supplemental Figure 4D). This correlated with an accumulation of p53 protein and induction of apoptosis in the embryonic stem cells in hypoxia (Supplemental Figure 4E). These data together demon- 

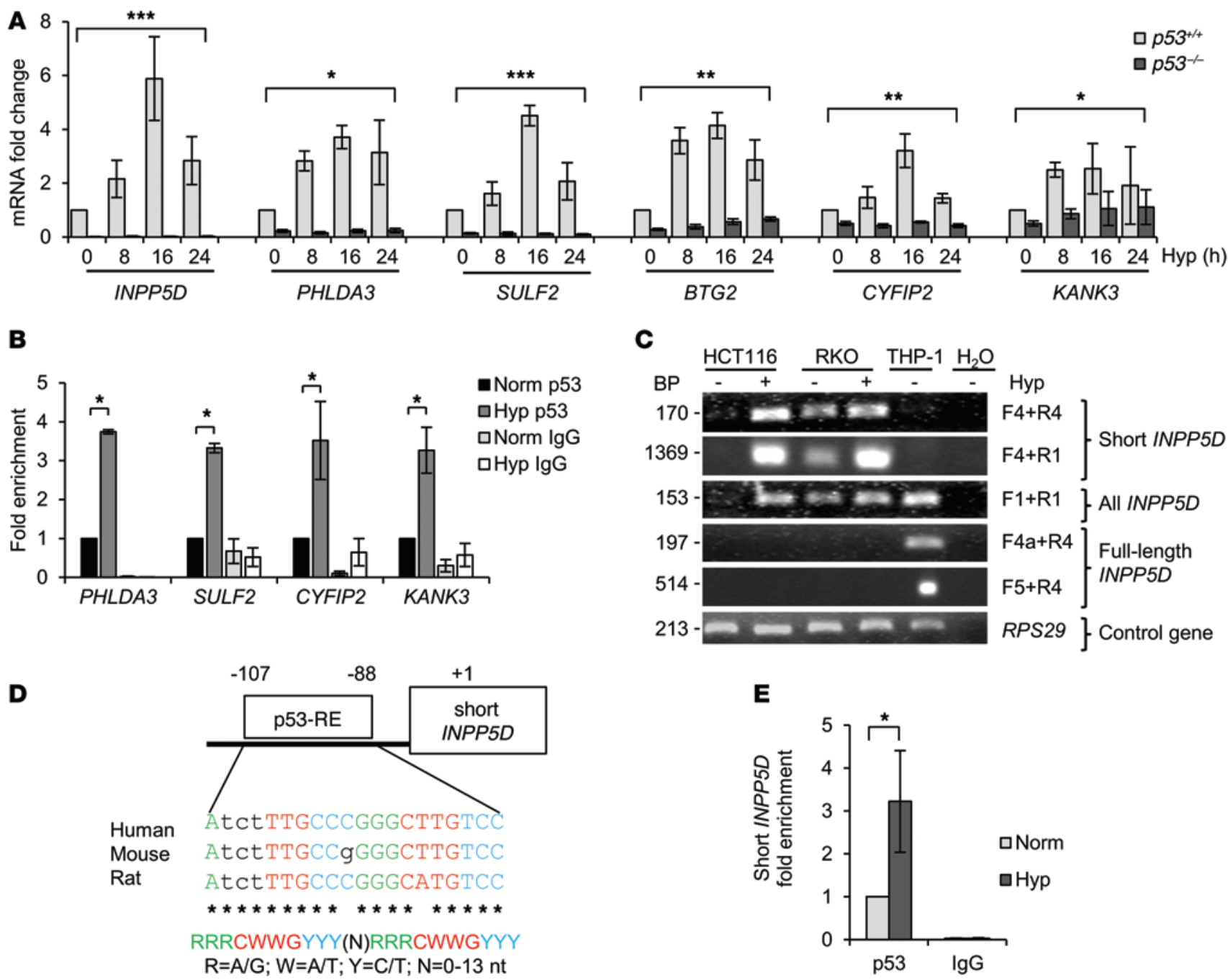

Figure 2. Transcriptional activity of $\mathbf{p} 53$ is required to induce expression of a specific group of genes in hypoxia. (A) Validation of $p 53$ targets by qPCR in $p 53^{+/+}$and $p 53^{-/-}$HCT116 cells exposed to hypoxia. The bar graph shows mean \pm SEM (combined $n=3,2$-way ANOVA test $\left[{ }^{*} P<0.05 ;{ }^{* *} P<0.01\right.$; $\left.{ }^{* *} P<0.001\right]$ ). (B) qPCR for $\mathrm{p} 53$ ChIP in HCT116 cells exposed to 8 hours of normoxia or hypoxia for the genes indicated. The bar graph shows mean \pm SEM (combined $n=3$, 2-way ANOVA test $[P<0.0001]$ followed by 2-tailed Student's $t$ test $\left[{ }^{*} P<0.05\right]$ ). (C) PCR for the INPP5D transcripts using a combination of primers (marked as arrows in Supplemental Figure 2A) specifically detecting the short INPP5D transcript (F4+R4 or F4+R1), all INPP5D transcripts (F1+R1), or the full-length INPP5D transcript (F4a+R4 or F5+R4) on the cDNA from HCT116 or RKO cells exposed to 16 hours of hypoxia. THP-1 cDNA was used as a control for the expression of the full-length INPP5D. RPS29 is shown as a control gene. (D) Alignment of the putative p53 RE of the short INPP5D in human, mouse, and rat. A consensus p53 RE is shown below the alignment. (E) qPCR for p53 ChIP at the putative p53 RE in the short INPP5D promoter in HCT116 cells exposed to 8 hours of normoxia or hypoxia. The bar graph shows mean \pm SEM (combined $n=3$, 2-tailed Student's $t$ test $\left.\left[{ }^{*} P<0.05\right]\right)$.

strate that in response to hypoxia p53 induces expression of a specific short INPP5D transcript, the expression of which was previously associated predominantly with embryonic stem cells.

The collective expression of hypoxic p53 targets is regulated in cancers and correlates with patient prognosis. To investigate the relevance of the p53 target genes induced in response to hypoxia in cancer patients, we analyzed all available Oncomine data sets using our validated p53 hypoxic targets: PHLDA3, INPP5D, SULF2, BTG2, CYFIP2, and KANK3. The underexpression of this group of genes correlated significantly with TP53 mutation in 9 studies (Supplemental Figure 5A); this represents a statistically significant enrichment with respect to association with any other mutation (hypergeometric test, $P=0.0279$ ). To further demon- strate the significance of this result, we defined an equally sized control group of known p53 target genes that are not induced by hypoxia (Figure 1C) but have well-characterized roles in DNA damage-induced apoptosis: PUMA, BID, PERP, BAX, PIG3, and NOXA (10). Although the analysis of this control group did identify 3 data sets where the underexpression of these genes was associated with TP53 mutation (Supplemental Figure 5B), this was not a statistically significant enrichment (hypergeometric test, $P=0.599)$. This led us to conclude that the p53-dependent genes identified here in vitro as hypoxia inducible are likely to be regulated by $\mathrm{p} 53$ in vivo. Moreover, the hypoxia-inducible group of genes (PHLDA3, INPP5D, SULF2, BTG2, CYFIP2, and KANK3) was a better predictor of p53 status than a control group of 


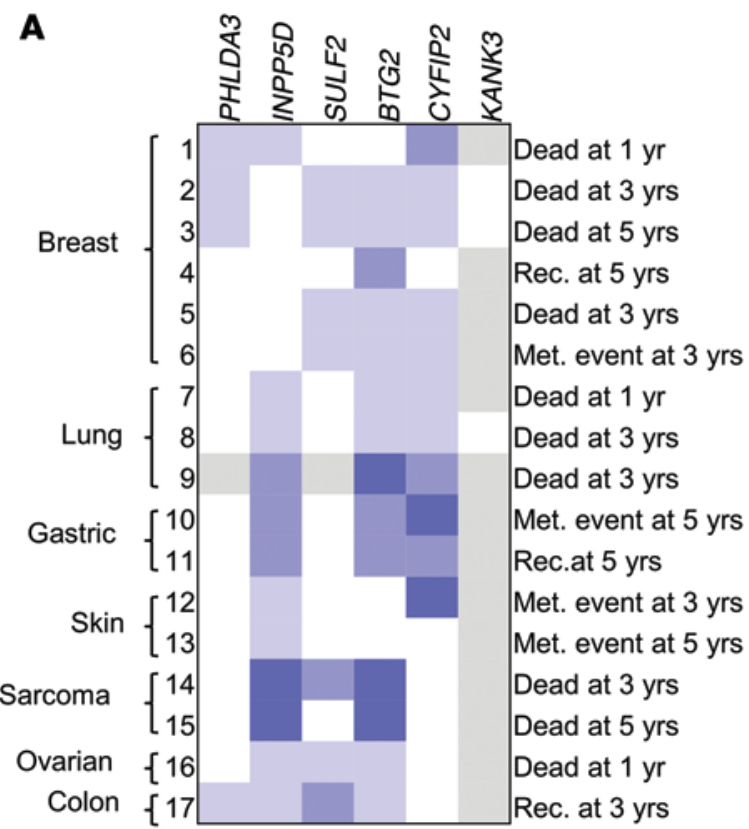

C

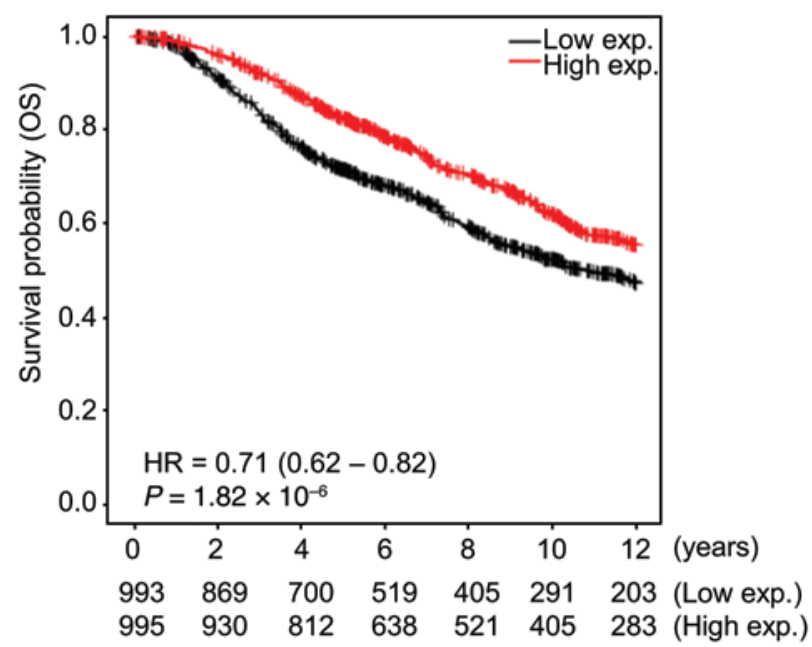

well-known p53 proapoptotic targets (PUMA, BID, PERP, BAX, $P I G 3$, and NOXA) induced in response to DNA damage.

We then tested whether collective expression of the group of genes induced by p53 under hypoxia (PHLDA3, INPP5D, SULF2, $B T G 2, C Y F I P 2$, and KANK3) was associated with any consistent clinical outcome in patients with cancer. An Oncomine search of all the available data for patients with cancer identified 17 data sets in which underexpression of this group of genes was significantly associated with a poor clinical outcome in patients with breast, lung, gastric, melanoma, sarcoma, ovarian, and colorectal cancers (Figure 3A). Conversely, no studies were found in which overexpression of this group of genes correlated with poor clinical outcome. The expression of these genes tested individually in Oncomine did not show any consistent pattern of their expression correlating with patient prognosis, suggesting that their concomitant regulation is the relevant factor for

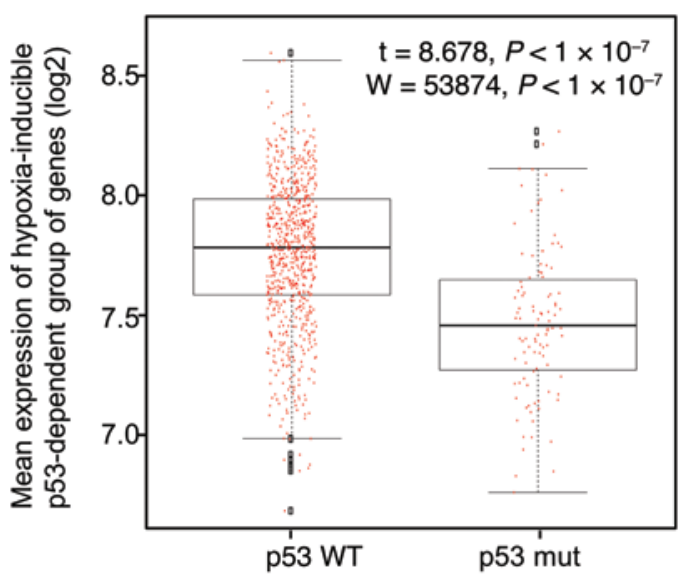

Figure 3. Underexpression of hypoxia-inducible proapoptotic p53 targets predicts p53 status and correlates with poor clinical outcome in patients with cancer. (A) Heat map showing a grouped expression of validated hypoxiainducible proapoptotic p53 targets (PHLDA3, INPP5D, SULF2, BTC2, CYFIP2, and $K A N K 3$ ) correlated with a clinical outcome in patients with cancer. Rows represent single data sets associated with the tested group of genes in the Oncomine database (described in the Supplemental Methods), with the cancer types indicated on the left and clinical outcome on the right. A significant fold change in gene expression $(P \leq 0.01)$ is shown in purple. Not significant and not tested (nt) expression of genes are shown in white and gray, respectively. rec., recurrence; met., metastatic. (B) Box plots showing the summary expression distribution for the $\mathrm{p53}$-dependent hypoxia-inducible group of genes from $\mathbf{A}$ in p53 WT and mutant (mut) breast cancer samples from the METABRIC breast cancer patient cohort (32). Red dots show the scores for individual patients, jittered horizontally. The bottom and top of each box represent the first and third quartiles, respectively. The line and band inside each box indicate median summary expression. The whiskers of the box indicate 1.5 times interquartile range. The mean expression of these genes was considered as the summary expression. Wilcoxon test and $t$ test results are provided on the plot. (C) A KaplanMeier plot showing meta-analysis for the overall survival (OS) and expression of hypoxia-inducible p53-dependent genes performed on the METABRIC breast cancer patient cohort. The total number of patients at risk at time points 0 to 12 years is shown below the $x$ axis. HR, hazard ratio.

clinical outcome. When we again compared the control group of well-characterized p53 proapoptotic genes (PUMA, BID, PERP, $B A X, P I G 3$, and NOXA), we found that only 4 data sets were significantly associated with their collective expression; notably, in all 4 cases, it was the overexpression, rather than underexpression, of these genes that correlated with poor clinical outcome (Supplemental Figure 5C).

Next, we carried out independent meta-analyses on publically available, large, and well-characterized breast cancer patient cohorts. The first analysis was performed on 6 curated retrospective breast cancer data sets deposited in the Gene Expression Omnibus repository, in which clinical information was published for a total of 1,050 cases (GSE6532KI, GSE6532GUY, GSE6532OXF, GSE9195, GSE1456, and GSE2034). Tests for heterogeneity showed that the 6 data sets could be merged safely $(P=0.785)$. The p53 status of these samples is 

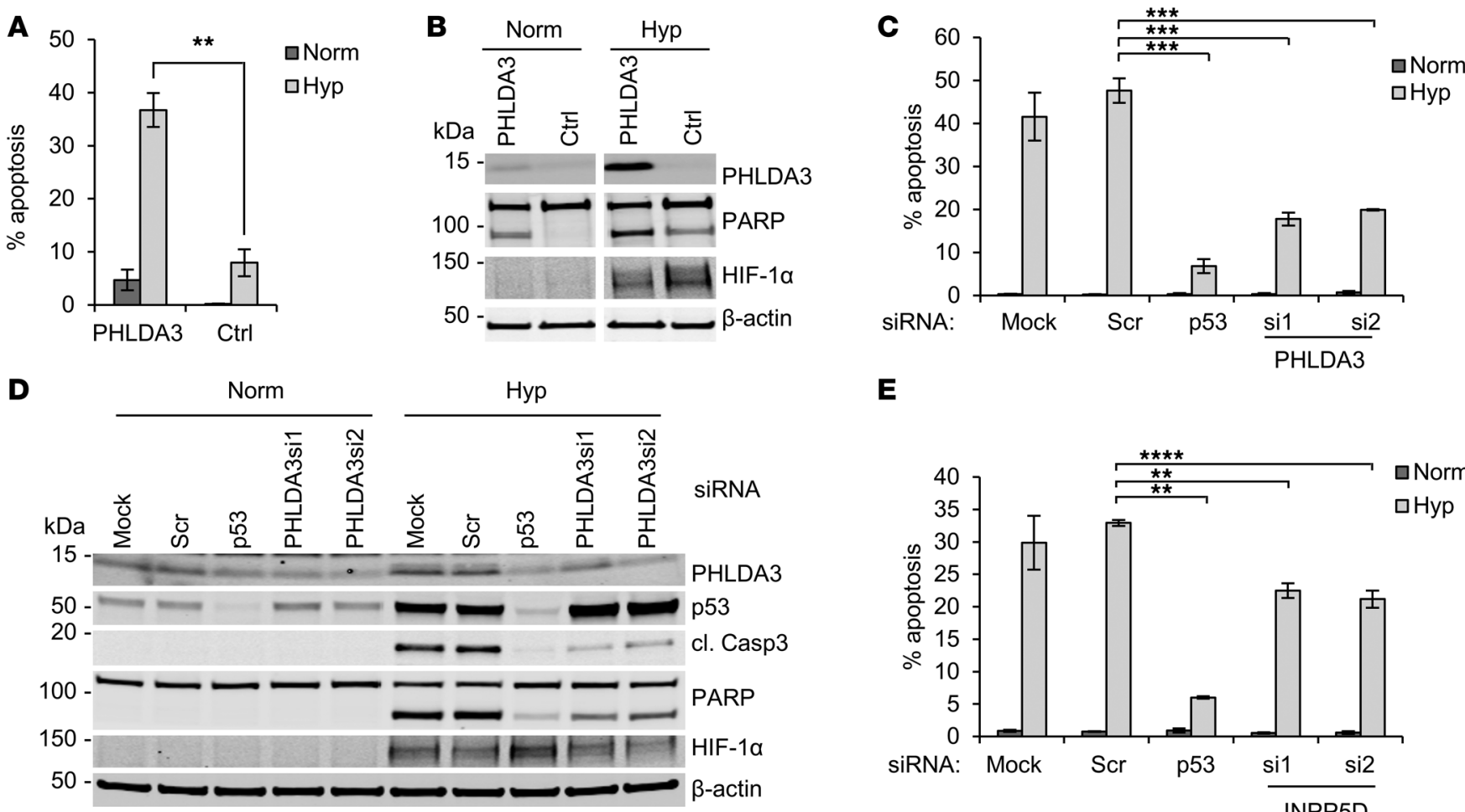

E
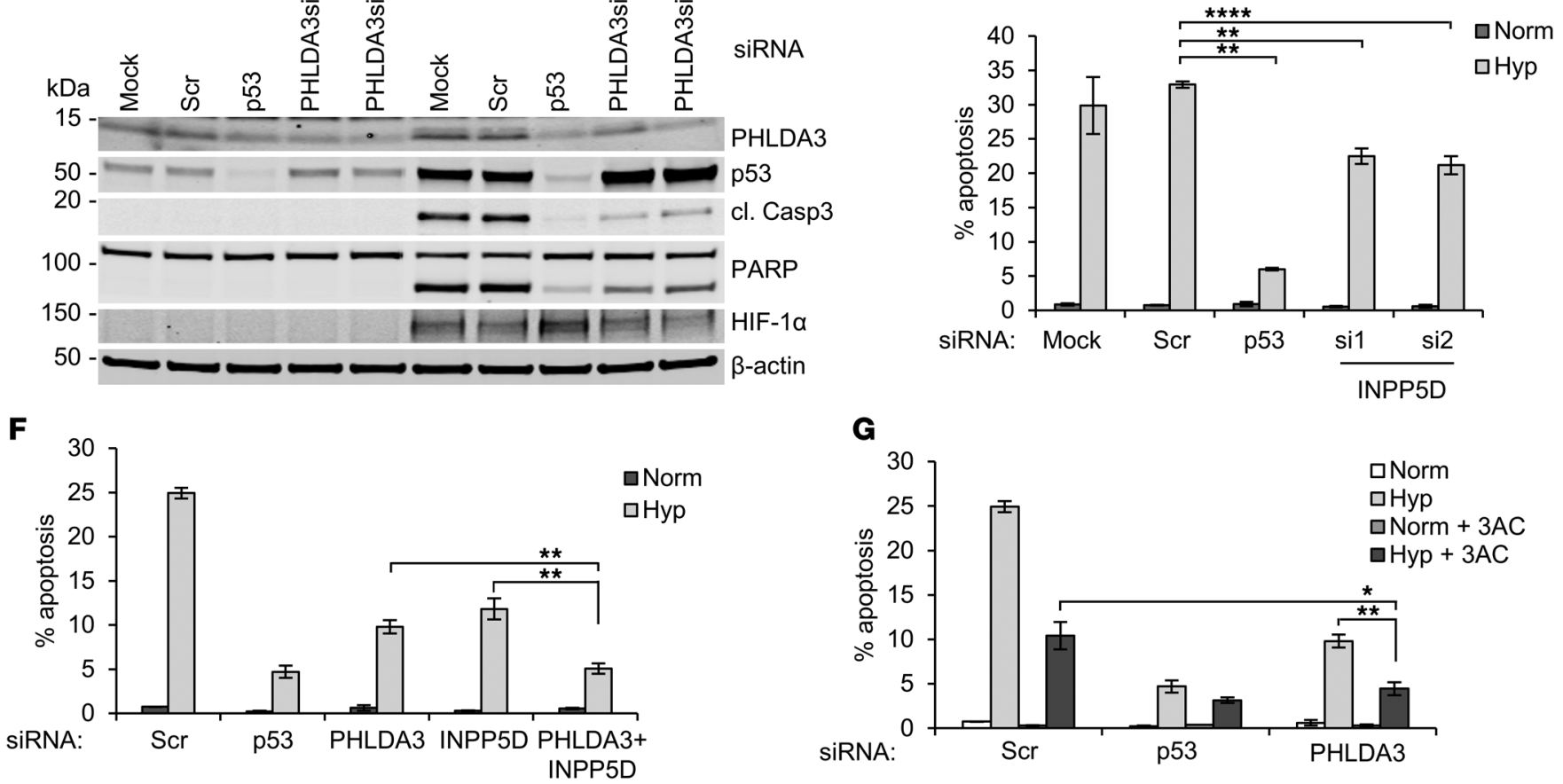

Figure 4. p53 targets PHLDA3 and INPP5D mediate apoptosis in hypoxia. (A and B) Apoptosis detected morphologically or by Western blotting for PARP cleavage, respectively, in H1299 cells transfected with 5xHRE-PHLDA3 and exposed to 24 hours of hypoxia. The bar graph shows mean \pm SEM $\left(n=3\right.$; 2-tailed Student's $t$ test [ $\left.\left.{ }^{* *} P<0.01\right]\right)$. For Western blotting, the lanes were run on the same gel but were noncontiguous. (C and $\left.\mathbf{D}\right)$ Apoptosis detected morphologically or by Western blotting for cleaved PARP or caspase-3 (cl. Casp3) in RKO cells treated with nonspecific (Scr), PHLDA3, or p53 siRNA and exposed to 18 hours of hypoxia or normoxia. The bar graph shows mean \pm SEM $(n=3,2$-way ANOVA test $[P<0.0001]$ followed by 2 -tailed Student's $t$ test $\left[{ }^{* *} P<0.01\right]$ ). (E and $\mathbf{F}$ ) Apoptosis detected in RKO cells treated with nonspecific, PHLDA3, INPP5D, or p53 siRNA as indicated and exposed to 14 hours of hypoxia or normoxia. The bar graphs show mean \pm SEM ( $n=3,2$-way ANOVA tests $[P<0.0001$ for both graphs] followed by 2-tailed Student's $t$ test [ ${ }^{* *} P<0.01$; $\left.\left.{ }^{* * *} P<0.0001\right]\right)$. (G) Apoptosis detected morphologically in RKO cells treated with nonspecific, p53, or PHLDA3 SiRNA and exposed to 14 hours of hypoxia or normoxia in the presence or absence of INPP5D/SHIP-1 inhibitor $3 A C$. The bar graph shows mean \pm SEM ( $n=3,2$-way ANOVA test $[P<0.0001]$ followed by 2-tailed Student's $t$ test $\left.\left[{ }^{*} P<0.05 ;{ }^{* *} P<0.01\right]\right)$.

unknown, and so we used a recently described p53 gene signature to determine the likely p53 status and then asked whether our group of genes was expressed in a p53-dependent manner (38). Using this approach, we found that our p53-dependent hypoxia-inducible group of genes was expressed at a significantly lower level in p53-mutated samples $\left(P<1 \times 10^{-7}\right.$; Supplemental Figure $6 \mathrm{~A})$. This provided independent validation for the conclusion reached through earlier Oncomine analysis, showing that the group of hypoxic p53 targets is significantly and consistently associated with $\mathrm{p} 53$ mutation status in vivo. Next, we found that underexpression of hypoxic p53 targets correlated with a lower recurrence-free survival, with a summary effect hazard ratio of 0.63 (95\% confidence limits of 0.42 and 0.96 ) in the breast cancer data sets (Supplemental Figure 6B). We then carried out a similar meta-analysis on the data from the recently published METABRIC study, which consists of a large cohort of patients with breast cancer, for a total of 2,000 human cancer samples (39). The p53 status in a subset of these samples was known, and we confirmed once more in an independent data set that lower expression of our hypoxia-inducible p53-dependent genes significantly correlated with p53 mutation status (Figure 3B). As expected, breast cancer subtypes associated with high rates of p53 mutations, such as basal-like cancers (40), showed the lowest expression of the group of genes (Supplemental Figure 7). 
A

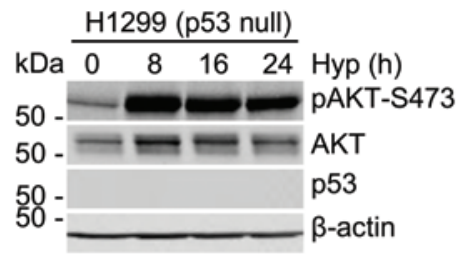

B

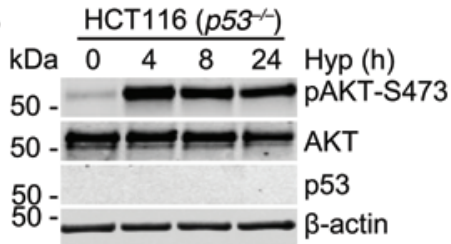

C

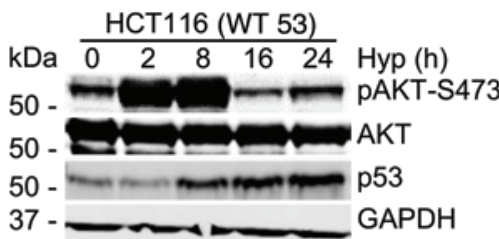
GAPDH
D

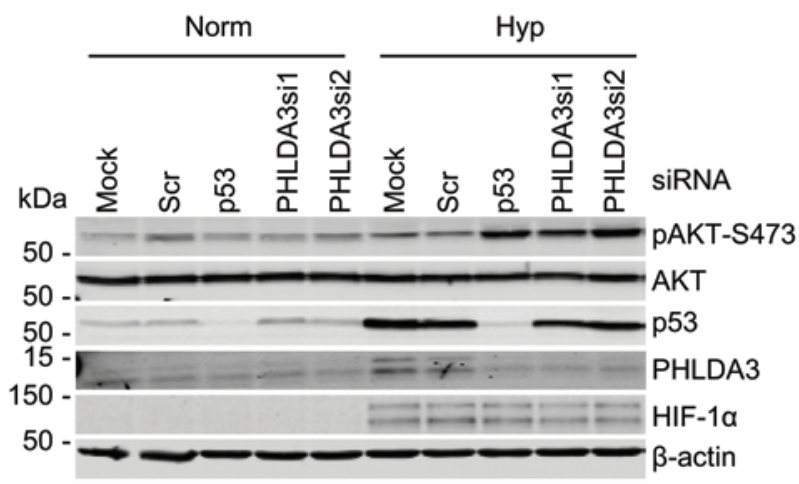

$\mathbf{F}$

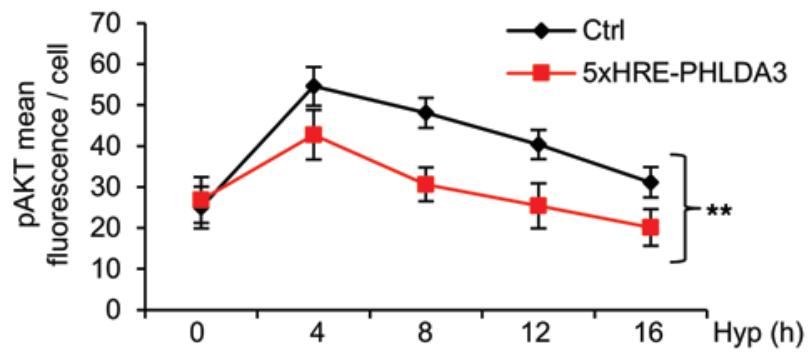

H

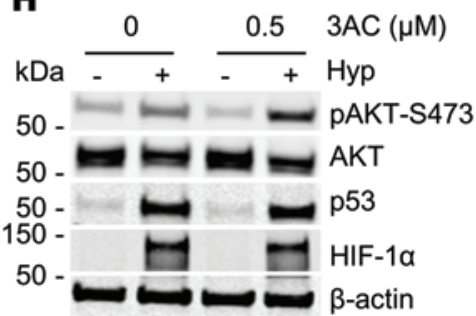

E
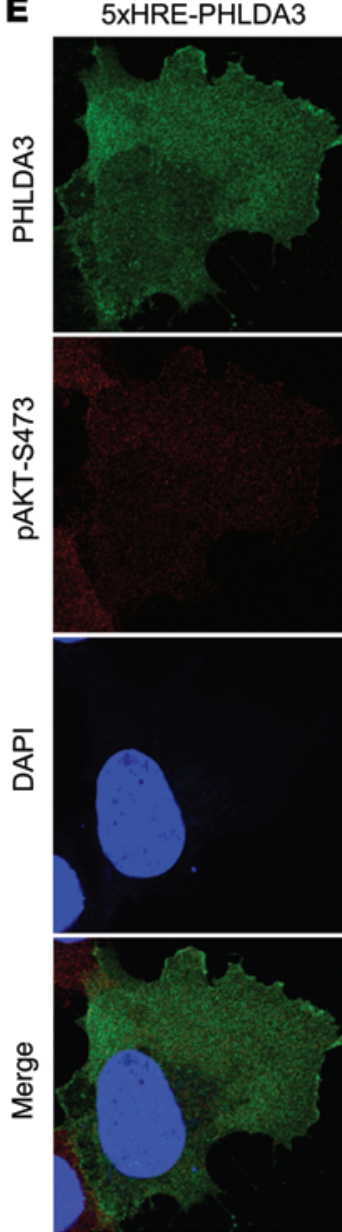

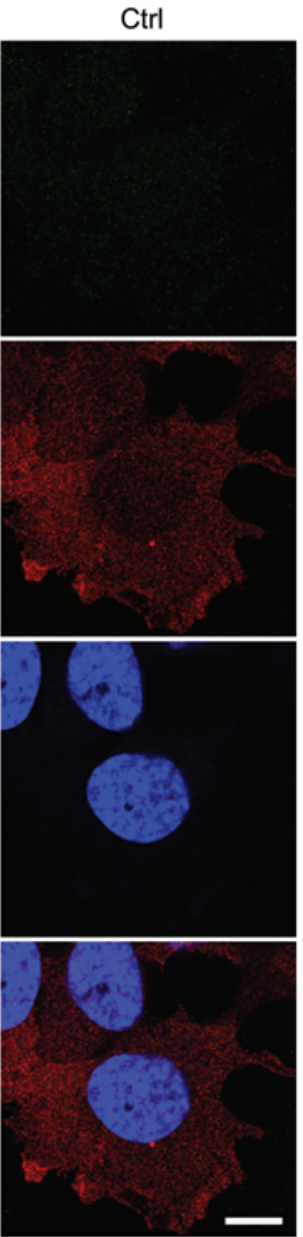

Figure 5. Hypoxia-induced activation of AKT is attenuated by p53/PHLDA3/SHIP-1 signaling. (A-C) Western blotting comparing AKT-S473 phosphorylation in H1299, HCT116 $\left(p 53^{--}\right)$, and WT HCT116 cells, respectively, exposed to hypoxia for the times indicated. (D) Western blotting for antibodies indicated in RKO cells transfected with either $\mathrm{p} 53$ or PHLDA3 siRNA and exposed to 8 hours of hypoxia. (E) Representative images of immunofluorescent costaining with anti-PHLDA3 (green) and anti-pAKT-S473 (red) antibodies in H1299 cells transfected with 5XHRE-PHLDA3 or control and exposed to hypoxia for 12 hours. Scale bar: $10 \mu \mathrm{m}$. (F) Hypoxic time course for the mean intensity of pAKT-S473 fluorescence per cell in control or PHLDA3-expressing cells from E. The plot shows mean $\pm \operatorname{SEM}\left(n=3,2\right.$-way ANOVA test $\left.\left[{ }^{* *} P<0.01\right]\right)$. (C) Western blotting in HCT116 cells exposed to 18 hours of normoxia or hypoxia in the presence or absence of SHIP-1 inhibitor, 3AC. (H) Western blotting in RKO cells exposed to 14 hours of normoxia or hypoxia in the presence or absence of $3 A C$.

This analysis also highlighted that these genes are expressed at higher levels in tumor tissue compared with that in normal tissue. Again, the lower expression of this group of genes correlated with poor patient outcome, as measured by overall survival over 12 years in the entire METABRIC cohort (Figure 3C).

PHLDA3 and INPP5D mediate p53-dependent apoptosis in hypoxia. Since the collective expression of the p53 target genes inducible in response to hypoxia significantly correlated with the clinical outcome of patients with cancer, we went on to investigate the function of these genes in hypoxia. Interestingly, two of the most highly induced p53 targets in hypoxia, PHLDA3 and INPP5D, were both previously shown to induce apoptosis through negative regulation of AKT signaling $(32,37,41)$. This led us to hypothesize that p53 can induce apoptosis in hypoxia via inhibition of AKT and that this is reliant on the coordinated functions of more than one target gene. We expressed a 5xHRE-PHLDA3 construct in p53-null H1299 cells and exposed them to hypoxia. As expected, PHLDA3 localized to the plasma membrane and significantly increased the percentage of apoptosis (Figure 4, A and B, and Supplemental Figure 8A). siRNAmediated knockdown of PHLDA3 significantly compromised hypoxia-induced apoptosis in RKO cells, although not to the same 

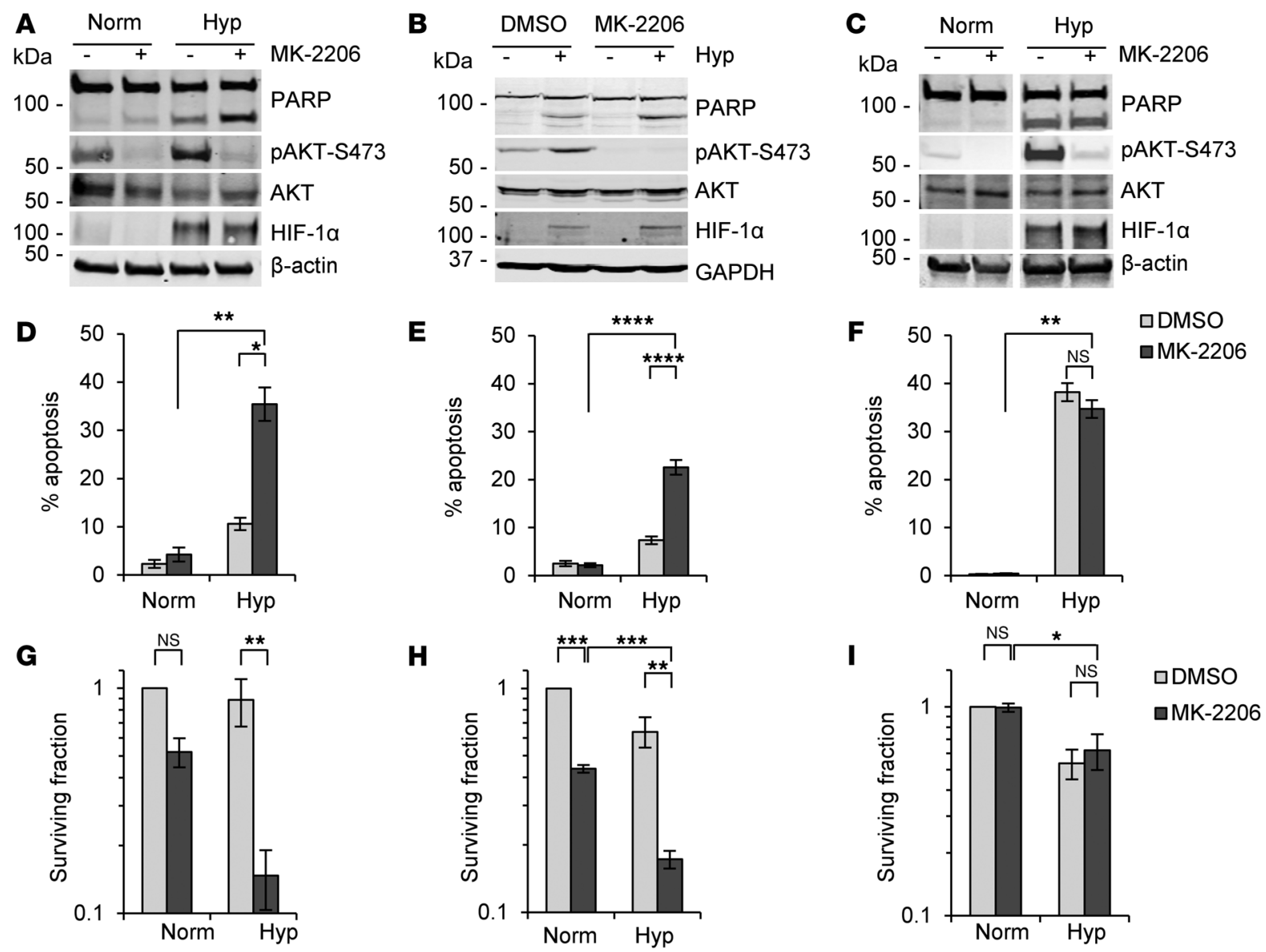

Figure 6. Pharmacological inhibition of AKT sensitizes p53-deficient hypoxic tumor cells. (A-C) Apoptosis detected by PARP cleavage in OE21, H1299, and HCT116 cells, respectively, exposed to 24 hours of hypoxia or normoxia in the presence of MK-2206 or DMSO. For Western blotting in C, the lanes were run on the same gel but were noncontiguous. (D-F) Apoptosis detected morphologically in OE21, H1299, and HCT116 cells, respectively, exposed to 24 hours of hypoxia or normoxia in the presence of MK-2206 or DMSO. (G-I) Colony survival assay in OE21, H1299, and HCT116 cells, respectively, exposed to 24 hours of hypoxia or normoxia in the presence of MK-2206 or DMSO. All the bar graphs show mean \pm SEM ( $n=3 ; 2$-tailed Student's $t$ test $\left[{ }^{*} P<0.05\right.$; ${ }^{*} P<0.01$; ${ }^{* *} P<0.001$; $\left.{ }^{* * *} P<0.0001\right]$ ).

extent as p53 siRNA knockdown (Figure 4, C and D). Likewise, siRNA knockdown of short INPP5D also significantly impaired hypoxia-induced apoptosis in these cells (Figure 4E). However, when both p53 targets, PHLDA3 and short INPP5D, were knocked down, the decrease in hypoxia-induced apoptosis was more profound than the levels of apoptosis observed with single knockdown of PHLDA3 or INPP5D alone and similar to the levels of apoptosis induced by p53-targeted siRNA, suggesting that these targets synergize in order to mediate apoptosis (Figure $4 \mathrm{~F}$ ). The efficiency of PHLDA3, p53, and INPP5D knockdown is quantified in Supplemental Figure 8, B-D. The proapoptotic function of the SHIP-1 protein (encoded by INPP5D) is reliant on its phosphatase domain, which is included in the short INPP5D transcript (37). Given that the phosphatase activity of SHIP-1 is essential for the induction of apoptosis, we investigated the SHIP-1-specific phosphatase domain inhibitor, 3AC (42). The use of $3 \mathrm{AC}$ decreased hypoxia-induced apoptosis in a dosedependent manner in a number of p53 WT cell lines (Supplemental Figure 8, E-G). In addition, when the SHIP-1 inhibitor, 3AC, was combined with PHLDA3 siRNA knockdown in RKO cells, we found that the combination reduced apoptosis further than $3 \mathrm{AC}$ combined with a nontargeting siRNA (Figure 4G). In summary, these data confirm that both p53 targets, PHLDA3 and short INPP5D, contribute to p53-dependent apoptosis in hypoxia.

Hypoxia-induced activation of AKT is sustained in the absence of $p 53$ function. In contrast to our hypothesis that $p 53$ induces apoptosis in hypoxia by inhibiting AKT signaling, previous studies have demonstrated that hypoxia activates AKT $(43,44)$. We also found that hypoxia potently induced phosphorylation of AKT at S473 (pAKT-S473). However, we noted that this activation of AKT was only sustained in p53-null or p53 mutant cells (HCT116 p53-- , H1299, OE21, or PSN1) compared with p53 WT cells (RKO or HCT116) (Figure 5, A-C, and Supplemental Figure 8, H-J). These data support our hypothesis that p53 may be negatively regulating AKT activation in hypoxia. In further support of this, siRNA-mediated depletion of endogenous PHLDA3 or p53 in RKO cells increased pAKT-S473 in hypoxia (Figure 5D). We also found that overexpression of PHLDA3 in H1299 cells significantly reduced hypoxic activation of AKT, as judged by the fluorescent intensity of pAKT-S473 staining in PHLDA3-expressing cells compared with that in PHLDA3negative cells (Figure 5, E and F). In addition, inhibition of 
A

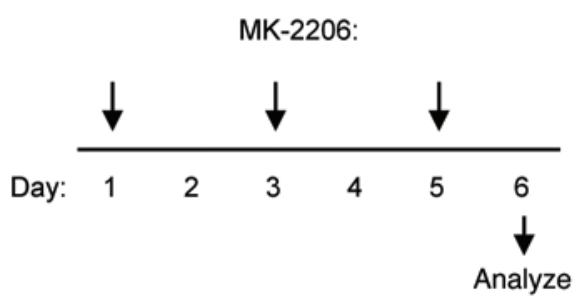

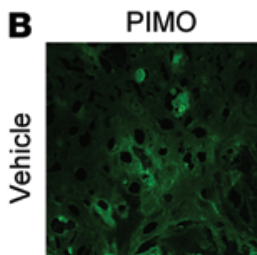

B

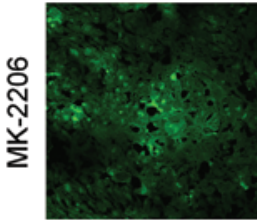

PAKT-S473
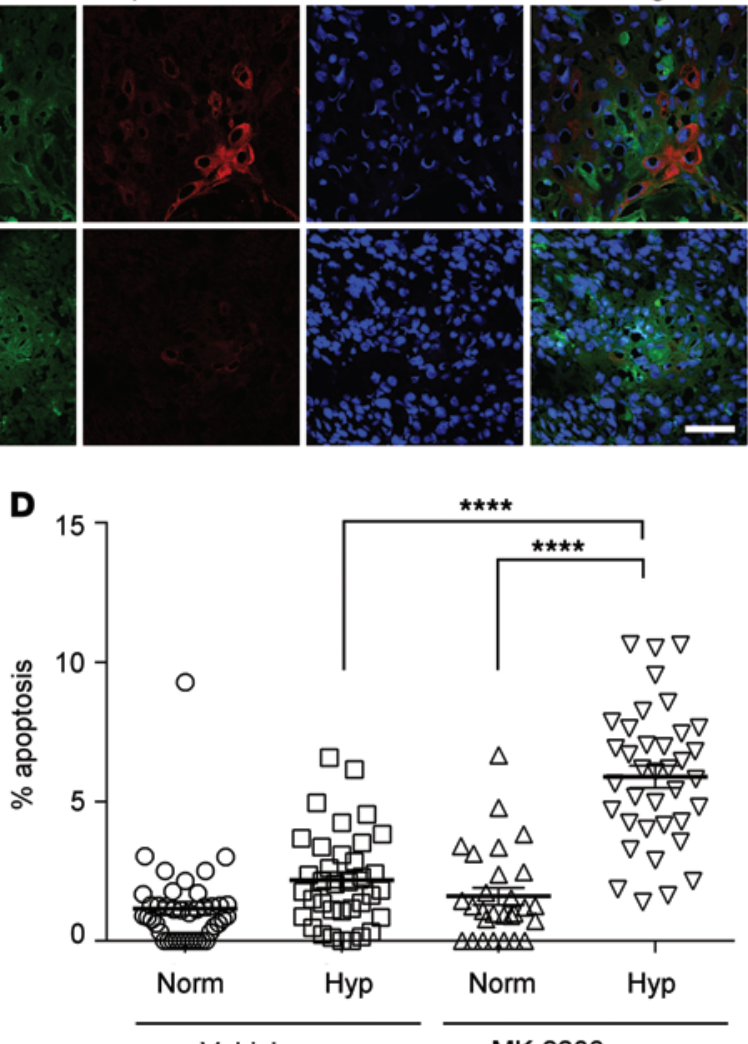

Vehicle
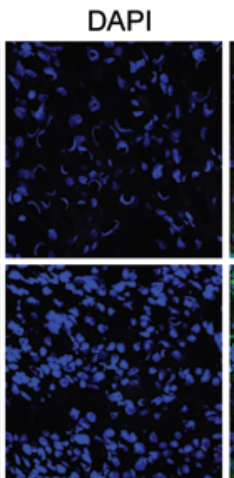

c CAIX
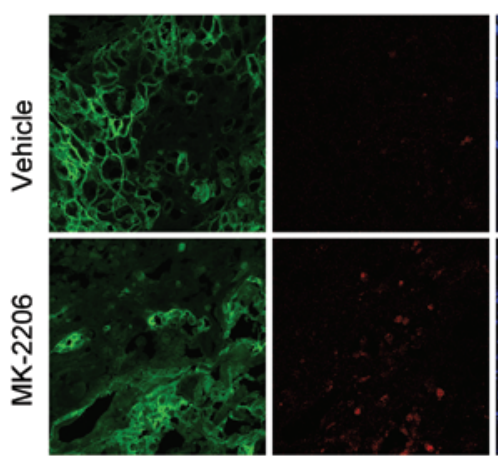

Cleaved
Casp 3

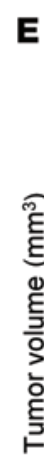

DAPI

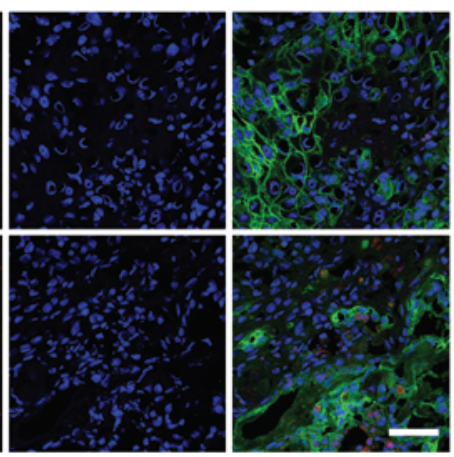

$\rightarrow$-Vehicle

$\longrightarrow$ MK-2206

$\rightarrow-V e h i c l e+I R$

-MK-2206+IR

$\mathbf{F}$

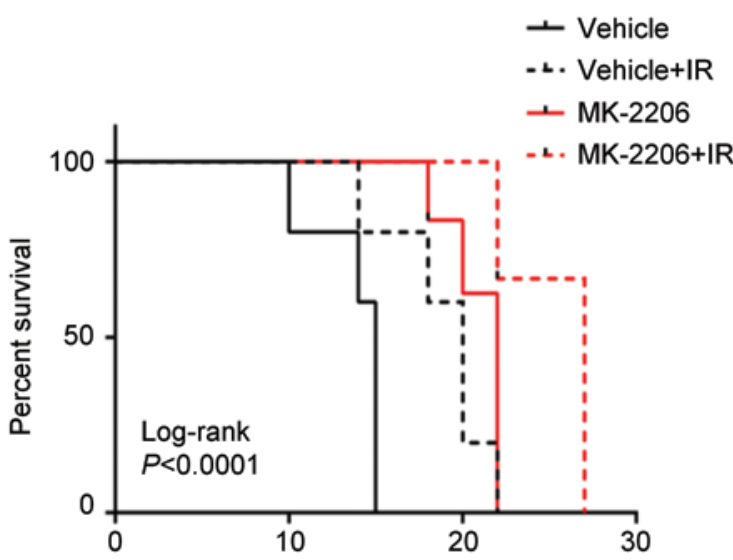

Days after randomization

MK IR

Figure 7. Pharmacological inhibition of AKT induces apoptosis in hypoxic regions of p53-deficient tumors and increases radiosensitivity. (A) MK-2206 treatment in OE21 xenografts. Mice were given 60 mg/kg MK-2206 or vehicle alone on 3 alternate days, and, 24 hours after the last dose, tumors were collected for analysis. (B) Representative images of colocalization of pAKT-S473 with hypoxic areas of OE21 xenografts stained with pimonidazole antibody (PIMO). Scale bar: $50 \mu \mathrm{m}$. (C) Representative images of colocalization of cleaved caspase-3 with hypoxic areas of $0 E 21$ xenografts stained with CAIX antibody. Scale bar: $50 \mu \mathrm{m}$. (D) Plots showing the percentage of apoptosis in either normoxic or hypoxic regions from all the regions analyzed. The bar represents the mean \pm SEM from 3 animals per each group (2-tailed Student's $t$ test [**** $P<0.0001]$ ). (E) 0E21 xenografts were grown in mice as in A, and a day after the last dose of MK-2206 or vehicle, tumors were treated with a single dose of IR (10 Gy). Tumor volumes were measured for 5 (vehicle), 5 (vehicle+IR), 6 (MK-2206), and 6 (MK-2206+IR) mice per each group, and mean \pm SEM is shown. (F) A Kaplan-Meier plot showing the survival probability of mice in each treatment group (log-rank [Mantel-Cox] test with $P<0.0001$ shown for all the curves compared together; comparisons of 2 single curves at the time were significant $[P<0.05]$ for all the combinations except the Vehicle+IR group versus MK-2206 group). 
A

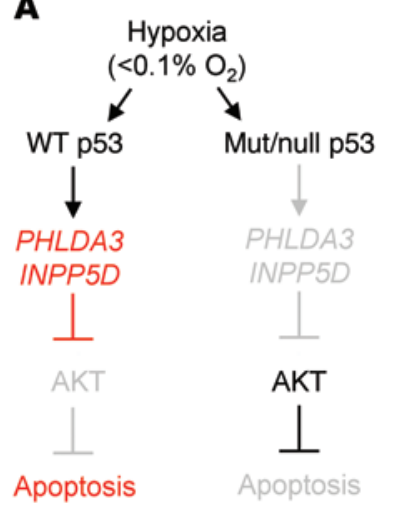

B

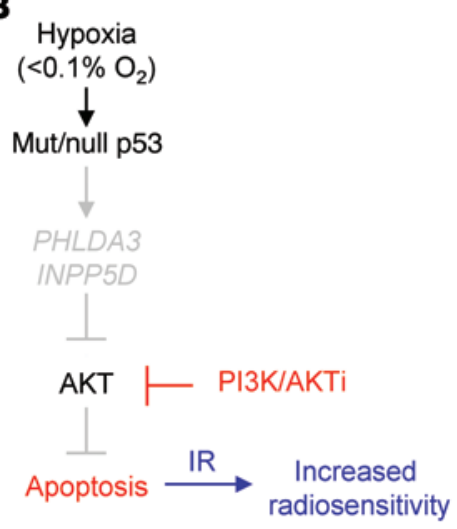

Figure 8. AKT inhibition radiosensitizes p53-deficient hypoxic tumors. (A) In response to hypoxia, p53 induces specific target genes, the role of which includes the induction of apoptosis. One of the mechanisms of apoptosis induction by $p 53$ in hypoxia is through the inhibition of AKT signaling via PHLDA3 and INPP5D. In the p53-deficient tumors, the expression of PHLDA3 and INPP5D is abrogated, which facilitates activation of AKT and consequently inhibition of apoptosis. (B) Targeting p53-deficient tumors with $\mathrm{PI} 3 \mathrm{~K} / \mathrm{AKT}$ inhibitors (PI3K/AKTi) is a promising strategy to restore the signaling of $p 53$ in the hypoxic cells and eliminate therapy-resistant tumor fractions.
SHIP-1 with 3AC increased pAKT-S473 in hypoxia in HCT116 and RKO cells (Figure 5, G and $\mathrm{H}$ ).

Inhibition of AKT increases apoptosis in p53-deficient hypoxic cells. Our data suggest that one of the functions of hypoxiainduced p53 is to restrict AKT signaling and drive cells into apoptosis. We asked whether the use of pharmacological inhibitors of AKT could be an effective approach to target hypoxic tumor cells. A panel of cells lines, expressing mutant p53 or not, were exposed to the allosteric AKT inhibitor MK-2206, and apoptosis assays were carried out. We observed a significant induction of apoptosis in cells (OE21, H1299, PSN1) treated with MK-2206 in combination with hypoxia but not with MK-2206 alone (Figure 6, A, B, D, and E, and Supplemental Figure 9, A-D). As expected, low levels of apoptosis were observed in these cells (p53 mutant/null) in response to hypoxia alone. This was also reflected in a difference in overall survival, as measured by clonogenic assay (Figure $6, \mathrm{G}$ and $\mathrm{H})$. In contrast, treatment with MK-2206 did not further increase hypoxia-induced apoptosis or affect overall survival in HCT116 cells expressing WT p53 (Figure 6, C, F, and I) or H1299 cells transfected with the HRE-p53 construct (Supplemental Figure 9, C and D). These data demonstrate that p53 inhibits AKT signaling in hypoxia and hypoxic cells that have lost $\mathrm{p} 53$ function are more sensitive to inhibition of AKT.

Inhibition of AKT increases apoptosis in hypoxic regions of p53deficient tumors and increases radiosensitivity. To validate these findings in vivo, we grew OE21 (p53p.s9ofs31X, c.269c $>$ T, c.270delc), PSN1 (p53 ${ }^{\mathrm{K} 132 \mathrm{Q}}$ ), and HCT116 ( $\mathrm{p5} 3^{\mathrm{WT}}$ ) cells as tumor xenografts and treated them with either MK-2206 or vehicle alone (Figure 7A; Supplemental Figure 10, A and B; Supplemental Figure 11A; and Supplemental Figure 12A). As expected, pAKT-S473 was induced in the pimonidazole-positive hypoxic regions of these tumors, and this was efficiently inhibited by treatment with MK-2206, as judged by immunofluorescent staining or Western blotting of harvested tumors (Figure 7B, Supplemental Figure 10, and Supplemental Figure 11B). Most importantly, we found that AKT inhibition significantly increased apoptosis in the hypoxic areas of the p53-deficient tumors (Figure 7, C and D, and Supplemental Figure 11, C and D) but not in those of the p53 WT tumors (Supplemental Figure 12, B and C), as determined by costaining of cleaved caspase- 3 and CAIX. These data support our hypothesis that the mechanism of p53-dependent hypoxia-induced apoptosis includes AKT inhibition and that this can be exploited in vivo through pharmacological inhibitors.
A decrease in the hypoxic fraction of solid tumors through increased apoptosis would be predicted to increase radiosensitivity. To test this, we chose to use an esophageal cancer model, due to the high rates of $\mathrm{p} 53$ mutation and the significant correlation between $\mathrm{p} 53$ status and patient prognosis in this cancer (45). OE21 xenografts were grown, and the mice were treated with either $60 \mathrm{mg} / \mathrm{kg}$ MK-2206 or vehicle on 3 alternate days, followed by a single dose of 10 Gy irradiation (IR) (Figure 7E). MK-2206 or IR alone delayed tumor growth from 100 to $250 \mathrm{~mm}^{3}$ for approximately 4 days in comparison to that in the vehicle-treated control group. However, the combined MK-2206 and IR treatment had a profound effect in delaying tumor growth from 100 to $250 \mathrm{~mm}^{3}$ for 12 days when compared with vehicle treatment or for 8 days when compared with either treatment with IR or MK-2206 alone (Figure $7 \mathrm{E}$ ). This was reflected by the significantly prolonged median survival of mice treated with the combined therapy in comparison to either treatment alone (Figure $7 F$ ). These data confirmed our hypothesis that the radiosensitizing potential of AKT inhibitors could in part be mediated through a decrease in the hypoxic fraction of tumors that have lost functional p53 (Figure 8).

\section{Discussion}

Our study demonstrates that p53 is transactivation competent in response to replication stress induced by hypoxia $\left(<0.1 \% \mathrm{O}_{2}\right)$. A validated subset of the genes identified was significantly and consistently associated with p53 mutation status and patient prognosis in several clinical cohorts of patients with cancer, which both confirms the p53 dependency and highlights the importance of the hypoxia-induced p53 response. Two of the genes identified (PHLDA3 and INPP5D) both inhibit AKT signaling and promote apoptosis in hypoxia. This suggests that tumors that have lost p53 function may be more sensitive to AKT inhibition, due to restoration of proapoptotic signaling in hypoxia (Figure 8). In support of this, we demonstrate increased apoptosis in hypoxia in response to an AKT inhibitor (MK-2206) in the absence of functional p53 both in vitro and in OE21 and PSN1 tumors in vivo. Most significantly, we found that esophageal p53-deficient tumors that had increased apoptosis specifically in hypoxic areas also showed an increased response to radiotherapy.

Recently a number of mouse models have been used to determine the mechanism of p53-mediated tumor suppression. These include the mutation of key residues such as K117/K161/K162 
(corresponding to K120/K164 in humans) or coordinated knockout of genes, such as PUMA, NOXA, and p21 $(24,25)$. These studies demonstrate that DNA damage-induced p53-dependent apoptosis mediated by the classic target genes is not required for tumor suppression. This is supported by our finding that the levels of PUMA, $B I D, P E R P, B A X, P I G 3$, and NOXA as a group do not predict patient outcome in clinical samples. Our study demonstrates that the hypoxia-induced transcriptional response of p53 uses alternative proapoptotic pathways, which substantially differ from those induced by genotoxic stress. Hypoxia-induced p53-mediated apoptosis could therefore be a crucial component of tumor suppression. In support of this hypothesis, some of the hypoxia-inducible p53 targets, such as $P H L D A 3$ and KANK3, have already been linked with p53-mediated tumor suppression $(23,46)$. By carrying out thorough and independent analyses of large clinical cohorts of breast and other cancers, we found that poor patient prognosis and progression of disease are significantly and consistently associated with diminished expression of the hypoxia-inducible p53-dependent group of genes, suggesting that their function is lost in aggressive cancers.

Previous studies have demonstrated that hypoxia-induced p53 is proapoptotic and suggest that this is the primary role for p53 in hypoxia $(11,18,21)$. The relevance of apoptosis was further supported by our finding that a number of the hypoxia-induced targets identified here had previously been described as proapoptotic, as opposed to having roles in alternative p53-mediated responses. However, it is probable that hypoxia-induced p53 has additional functions, i.e., other than driving cells into apoptosis. For example, it is well known that cancer cells have increased glycolytic flux and suppressed oxidative phosphorylation, even in the presence of oxygen, and recently, the tumor-suppressive function of p53 was attributed to inhibition of glycolytic flux through induction of TIGAR (24). Interestingly, in hypoxia, TIGAR has been showed to protect from cell death via regulation of mitochondrial reactive oxygen species (47). Therefore, it would be interesting to investigate whether any of the targets identified in this study have roles in regulating cell metabolism under hypoxic conditions and whether this could in turn play a role in tumor suppression.

INPP5D, which encodes SHIP-1, specifically converts phosphatidylinositol- $(3,4,5)$-trisphosphate $\left[\mathrm{PI}(3,4,5) \mathrm{P}_{3}\right]$ to $\mathrm{PI}(3,4)$ $\mathrm{P}_{2}$ and therefore depletes the pool of PI3K-generated PI $(3,4,5) \mathrm{P}_{3}$ at the plasma membrane, resulting in impaired AKT activation (41). However, AKT can be also recruited to the plasma membrane by $\mathrm{PI}(3,4) \mathrm{P}_{2}$ as well as $\mathrm{PI}(3,4,5) \mathrm{P}_{3}$. Therefore, it is significant that hypoxia-induced p53 also transactivates PHLDA3, which, through its pleckstrin homology domain, can bind both $\operatorname{PI}(3,4,5)$ $\mathrm{P}_{3}$ and $\mathrm{PI}(3,4) \mathrm{P}_{2}$, leading to efficient inactivation of AKT $(32,48)$. This confirms that one of the principle roles of hypoxia-induced p53 is to restrict protumorigenic PI3K/AKT signaling.

AKT activation in hypoxic regions of breast tumors has been linked with poor patient prognosis (49). One of the known functions of AKT is to block apoptosis through phosphorylation of multiple targets, for example, caspase-3/9 or BAD (50). Therefore, inhibition of AKT is a promising strategy to eliminate cancer cells via reactivation of apoptosis. Many PI3K/AKT inhibitors are currently undergoing clinical trials, yet surprisingly, the available clinical data show little evidence for single-agent activity $(51,52)$. However, several studies describe promising antitumor activity of
PI3K/AKT inhibitors when combined with radiotherapy (53-57). AKT inhibition has also been demonstrated to improve radiosensitivity through normalization of the tumor vasculature (58). Our study, for the first time to our knowledge, demonstrates that inhibition of AKT in hypoxia may have a different effect with respect to p53 status and suggests that patients with cancer stratified for high tumor hypoxia and loss of p53 may get the most benefit from combined radiotherapy and AKT inhibition. The group of 6 genes identified and validated in this study offers a promising candidate biomarker to identify patients who could benefit from this approach. In summary, by fully characterizing the p53 transcriptional response to hypoxia, we have identified a stress-specific proapoptotic response, which could be exploited therapeutically and is relevant to patient prognosis.

\section{Methods}

Cell lines and plasmid transfection. RKO and HCT116 (colorectal carcinoma), H1299 (non-small-cell lung carcinoma), OE21 (esophageal squamous carcinoma), PSN1 (pancreatic adenocarcinoma), WI38 (lung fibroblasts), CCE (mouse embryonic stem cells), and THP-1 (acute monocytic leukemia) cell lines and plasmid transfection procedures are described in the Supplemental Methods.

Hypoxia/drug treatment. A Bactron chamber (Shel Lab) was used for studies at $<0.1 \% \mathrm{O}_{2}$ and an in vivo 400 chamber (Baker Ruskinn) was used for $2 \% \mathrm{O}_{2}$. All hypoxic treatments were performed at $<0.1 \%$ $\mathrm{O}_{2}$ unless indicated otherwise. The following drug concentrations were used: $25 \mu \mathrm{M}$ etoposide (Sigma-Aldrich); $1 \mu \mathrm{M}$ MK-2206 (Selleck Chemicals); and 0.5-1 $\mu \mathrm{M}$ 3AC (Calbiochem).

Immunoblotting. Both adherent and detached cells were collected and lysed in SDS lysis buffer (10 mM Tris-Cl, pH 7.5, $0.1 \mathrm{mM}$ EDTA, $0.1 \mathrm{mM}$ EGTA, 0.5\% SDS, $0.1 \mathrm{mM} \beta$-mercaptoethanol, protease/phosphatase inhibitors). The following antibodies were used: p53 (DO-I, Santa Cruz), p53 (C-19, Santa Cruz), $\beta$-actin (AC-15, Santa Cruz), p53 (1C12, Cell Signaling Technology), p53-S15 (9284, Cell Signaling Technology), PARP (9542, Cell Signaling Technology), cleaved caspase-3 (9661, Cell Signaling Technology), pAKT-S473 (4060, Cell Signaling Technology), AKT (4691, Cell Signaling Technology), PHLDA3 (ab22822, Abcam), $\gamma$ H2AX (JBW301, Upstate-Millipore), H2AX (DR1016, Calbiochem), HIF-1 $\alpha$ (610958, BD Biosciences), and GAPDH (6C5, Novus Biologicals). The Odyssey infrared system (LI-COR) was used to image Western blots.

siRNA knockdown. RKO cells were transfected using DharmaFECT 1 reagent (Thermo Fisher Scientific). siRNA duplexes were used at $50 \mathrm{nM}$, and 24 hours after knockdown, cells were replated to be used in assays up to 72 hours after transfection. siRNA sequences are listed in the Supplemental Methods.

Apoptosis detection. Apoptosis by nuclear morphology was assessed in combined adherent and detached cells fixed in $4 \%$ paraformaldehyde. Apoptosis was measured as the percentage of cells with fragmented DNA per 10 fields in every treatment. Graphs show mean values \pm SEM from 3 independent experiments.

Colony survival assay. H1299, OE21, and HCT116 cells were treated either with DMSO or $1 \mu \mathrm{M}$ MK-2206 and exposed to 24 hours of hypoxia or normoxia. Subsequently, all cells were placed in a normoxic incubator and left for 9 days to form colonies, which were visualized by crystal violet staining. Graphs show mean values \pm SEM from 3 independent experiments. 
Immunofluorescence. Cells were grown on glass coverslips and stained as previously described (59). The following antibodies were used: PHLDA3 (ab22822, Abcam) and pAKT-S473 (4060, Cell Signaling Technology). A LSM780 confocal microscope (Carl Zeiss Microscopy Ltd) was used. The fluorescent intensity of pAKT-S473 per cell was measured from $\geq 15$ randomly selected cells imaged for each condition and was plotted as a mean of intensities \pm SEM from 3 independent experiments.

RNA extraction, microarray analysis, and qPCR. RNA was prepared using TRIzol (Invitrogen/Life Technologies). Gene expression array analysis was carried out using Agilent Sure Print HD arrays by Oxford Gene Technology. Differential gene expression regulated by p53 in hypoxia $\left(<0.1 \% \mathrm{O}_{2}\right)$ or in normoxia $\left(21 \% \mathrm{O}_{2}\right)$ was calculated as a $\log _{2}\left(\mathrm{p} 53 / \mathrm{p} 53^{175}\right)$ of intensities, and $t$ test with Benjamini and Hochberg $P$ value adjustment was applied (60). For qPCR expression analysis, cDNA was reverse transcribed from total RNA using the Verso Kit (Thermo Scientific). qPCR was performed using the SYBR Green PCR Master Mix Kit (Applied Biosystems) in a 7500 FAST Real-Time PCR thermocycler with v2.0.5 software (Applied Biosystems). Sequences of qPCR primers are shown in the Supplemental Methods. mRNA fold change was calculated using a $2^{-\Delta \Delta C t}$ method in relation to the $18 \mathrm{~S}$ reference gene. The qPCR graphs show the mean \pm SEM of 3 biological replicates.

ChIP. ChIP was carried out on HCT116 cells as described previously (7). Combined p53 antibodies (DO-I and FL-393, Santa Cruz) or combined nonspecific mouse and rabbit control IgGs (Cell Signaling Technology) were used for immunoprecipitation. For the qPCR, input samples were used at 100-fold dilution and ChIP samples were used at 6-fold dilution. Fold enrichment at specific promoter sites was calculated in relation to the input samples. Primers used for qPCR are listed in the Supplemental Methods.

Analysis of human tumor samples. The methods, selection, and curation of data sets for the meta-analysis based on Gene Expression Omnibus cohorts have been described previously (4). Briefly, the prognostic meta-analysis was carried out using the rmeta R package (http:// cran.r-project.org/). A fixed-effect model was used. The log hazard ratios and standard errors were obtained for each study from Cox proportional hazards regression. Study results were combined using the generic inverse-variance method. The variance was estimated as the square of the standard errors; this follows a $\chi^{2}$ distribution, with approximately $k-1$ degrees of freedom ( $k=$ number of studies). When groups of genes were considered, summary expression was estimated both as mean and median expression. In data sets in which p53 mutation status was not known, the p53 signature and related model were used to predict p53 mutation status as described previously (38). METABRIC data (39) were stratified by median expression of the gene signature to achieve balanced groups, and a Cox proportional hazards model was used to test equality of the survival distributions.

Oncomine analysis. Oncomine (https://www.oncomine.org/, research edition 4.4.3) results were generated using an odds ratio 2.0, $P$ value of 0.01 , and mRNA as a data type. No other filtering on data sets was applied, so the whole Oncomine cohort was used.

In vivo experiments. All animal procedures were performed in accordance with current United Kingdom legislation. Female athymic nude mice (BALB/c nude) (Harlan) were injected s.c. in the flank with $5 \times 10^{6}$ OE2 1 cells, $5 \times 10^{6}$ HCT116 cells, or $1 \times 10^{6} \mathrm{PSN}-1$ human pancreatic ductal carcinoma cells, the latter ones with $4 \times 10^{6}$ LTC-14 stellate cells.
Animals were randomized, and treatments were started when tumor volumes reached $100 \mathrm{~mm}^{3}$ (volume $=$ height $\times$ depth $\times$ width $\times \pi / 6$ ). Animal groups received either vehicle ( $25 \%$ cyclodextrin) or $60 \mathrm{mg} / \mathrm{kg}$ MK-2206 s.c. (PSN1 and HCT116) or orally (OE21) on 3 alternate days. Twenty-four hours after the last dose, tumors were harvested and snap frozen. Tumors embedded in OCT were sectioned $(5 \mu \mathrm{m})$ and immunofluorescence stained for hypoxia with CAIX antibody (M75 mouse monoclonal; ref. 61) or hypoxyprobe-1 pimonidazole antibody (clone 4.3.11.3, Hypoxyprobe) and with cleaved caspase-3 antibody (9661, Cell Signaling Technology). Apoptotic cells were counted within and outside of hypoxic regions. LSM780 software (Carl Zeiss) was used to determine the average of a single cell's surface area, and area of apoptotic cells (expressed as percentage) in whole hypoxic or normoxic regions was calculated from the following formula: number of apoptotic cells $\times$ average area of a single cell $\times 100 /$ area of total region measured. Images were taken from at least 2 tumors treated with vehicle and 3 tumors treated with MK-2206. Graphs show the percentage of apoptotic cells from all normoxic or hypoxic regions and treatments analyzed in every tumor. The mean \pm SEM is indicated by the bar. For the tumor growth curves, animals were s.c. injected with OE21 cells and randomized when average tumor volumes reached $100 \mathrm{~mm}^{3}$. Animals received vehicle or $60 \mathrm{mg} / \mathrm{kg}$ of MK-2206 on 3 alternate days, as above, and 24 hours after the last dose, the tumors in half of the animals in each treatment group were irradiated with $10 \mathrm{~Gy}$. Tumors were measured regularly, and tumor growth was plotted as a mean of tumor volumes \pm SEM. The number of mice for each treatment is as follows: 5 for vehicle, 5 for vehicle plus IR, 6 for MK-2206, and 6 for MK-2206 plus IR.

Statistics. Statistical tests other than the ones on clinical samples were performed using GraphPad Prism 6 software (GraphPad Software Inc.) and are indicated in figure legends for particular experiments. These included 2-way ANOVA tests, 2-tailed Student's $t$ test, and log-rank (Mantel-Cox) tests. $P$ values of less than 0.05 were considered significant.

Study approval. All animal experiments were approved by the University of Oxford Biomedical Services Ethical Review Committee. Ethical approval for the analysis of human breast cancer samples was obtained from the Molecular Taxonomy of Breast Cancer International Consortium.

\section{Acknowledgments}

We thank Adrian Harris, Amato Giaccia, Anderson Ryan, and Emmanouil Fokas for critical reading of the manuscript; Rieko Ohki for reagents; and Sally Hill, Karla Watson, and Mike Stratford for technical assistance. K.B. Leszczynska and E.M. Hammond are supported by Cancer Research UK (grant awarded to E.M. Hammond). Additional funds were provided by the Royal Society (awarded to E.M. Hammond) and European Union FP7 program (awarded to F.M. Buffa). This study makes use of data generated by the Molecular Taxonomy of Breast Cancer International Consortium, which was funded by Cancer Research UK and the British Columbia Cancer Agency Branch.

Address correspondence to: Ester M. Hammond, Cancer Research UK and Medical Research Council Oxford Institute for Radiation Oncology, Department of Oncology, University of Oxford, Old Road Campus Research Building, Oxford, OX3 7DQ. Phone: 01865.617320; E-mail: Ester.Hammond@oncology.ox.ac.uk. 
1. McKeown SR. Defining normoxia, physoxia and hypoxia in tumours-implications for treatment response. Br J Radiol. 2014;87(1035):20130676

2. Begg AC, Stewart FA, Vens C. Strategies to improve radiotherapy with targeted drugs. Nat Rev Cancer. 2011;11(4):239-253.

3. Hockel M, Vaupel P. Tumor hypoxia: definitions and current clinical, biologic, and molecular aspects. J Natl Cancer Inst. 2001;93(4):266-276.

4. Buffa FM, Harris AL, West CM, Miller CJ. Large meta-analysis of multiple cancers reveals a common, compact and highly prognostic hypoxia metagene. Br J Cancer. 2010;102(2):428-435.

5. Chitneni SK, Palmer GM, Zalutsky MR, Dewhirst MW. Molecular imaging of hypoxia. J Nucl Med. 2011;52(2):165-168.

6. Vaupel P, Kallinowski F, Okunieff P. Blood flow, oxygen and nutrient supply, and metabolic microenvironment of human tumors: a review. Cancer Res. 1989;49(23):6449-6465.

7. Olcina MM, et al. Replication stress and chromatin context link ATM activation to a role in DNA replication. Mol Cell. 2013;52(5):758-766.

8. Mazouzi A, Velimezi G, Loizou JI. DNA replication stress: Causes, resolution and disease. Exp Cell Res. 2014;329(1):85-93

9. Hammond EM, Denko NC, Dorie MJ, Abraham RT, Giaccia AJ. Hypoxia links ATR and p53 through replication arrest. Mol Cell Biol. 2002;22(6):1834-1843.

10. Brady CA, Attardi LD. p53 at a glance. J Cell Sci. 2010;123(pt 15):2527-2532.

11. Graeber TG, et al. Hypoxia-mediated selection of cells with diminished apoptotic potential in solid tumours. Nature. 1996;379(6560):88-91.

12. Brosh $\mathrm{R}$, Rotter $\mathrm{V}$. When mutants gain new powers: news from the mutant $\mathrm{p} 53$ field. Nat Rev Cancer. 2009;9(10):701-713.

13. Hammond EM, Dorie MJ, Giaccia AJ. ATR/ATM targets are phosphorylated by ATR in response to hypoxia and ATM in response to reoxygenation. J Biol Chem. 2003;278(14):12207-12213.

14. Alarcon R, Koumenis C, Geyer RK, Maki CG, Giaccia AJ. Hypoxia induces p53 accumulation through MDM2 down-regulation and inhibition of E6-mediated degradation. Cancer Res. 1999;59(24):6046-6051.

15. Lee JH, et al. Hypoxia activates tumor suppressor p53 by inducing ATR-Chk1 kinase cascade-mediated phosphorylation and consequent 14-3-3 $\gamma$ inactivation of MDMX protein. J Biol Chem. 2012;287(25):20898-20903.

16. Hammond EM, Giaccia AJ. The role of p53 in hypoxia-induced apoptosis. Biochem Biophys Res Commun. 2005;331(3):718-725.

17. Soengas MS, et al. Apaf-1 and caspase-9 in p53-dependent apoptosis and tumor inhibition. Science. 1999;284(5411):156-159.

18. Hammond EM, et al. Genome-wide analysis of 533 under hypoxic conditions. Mol Cell Biol. 2006;26(9):3492-3504.

19. Johnson TM, Hammond EM, Giaccia A, Attardi LD. The p53QS transactivation-deficient mutant shows stress-specific apoptotic activity and induces embryonic lethality. Nat Genet. 2005;37(2):145-152.

20. Li YZ, Lu DY, Tan WQ, Wang JX, Li PF. p53 ini- tiates apoptosis by transcriptionally targeting the antiapoptotic protein ARC. Mol Cell Biol. 2008;28(2):564-574.

21. Koumenis $\mathrm{C}$, et al. Regulation of $\mathrm{p} 53$ by hypoxia: dissociation of transcriptional repression and apoptosis from p53-dependent transactivation. Mol Cell Biol. 2001;21(4):1297-1310.

22. Fei $\mathrm{P}$, et al. Bnip3L is induced by $\mathrm{p} 53$ under hypoxia, and its knockdown promotes tumo growth. Cancer Cell. 2004;6(6):597-609.

23. Brady CA, et al. Distinct p53 transcriptional programs dictate acute DNA-damage responses and tumor suppression. Cell.2011;145(4):571-583.

24. Li T, et al. Tumor suppression in the absence of p53-mediated cell-cycle arrest, apoptosis, and senescence. Cell. 2012;149(6):1269-1283.

25. Valente LJ, et al. p53 efficiently suppresses tumor development in the complete absence of its cellcycle inhibitory and proapoptotic effectors $\mathrm{p} 21$, Puma, and Noxa. Cell Rep. 2013;3(5):1339-1345.

26. Kenzelmann Broz D, et al. Global genomic profiling reveals an extensive p53-regulated autophagy program contributing to key p53 responses. Genes Dev. 2013;27(9):1016-1031.

27. Mellert HS, et al. Deacetylation of the DNA-binding domain regulates p53-mediated apoptosis. J Biol Chem. 2011;286(6):4264-4270.

28. Adamsen BL, Kravik KL, Clausen OP, De Angelis PM. Apoptosis, cell cycle progression and gene expression in TP53-depleted HCT116 colon cancer cells in response to short-term 5 -fluorouracil treatment. Int JOncol. 2007;31(6):1491-1500.

29. Hong JW, Ryu MS, Lim IK. Phosphorylation of serine 147 of tis21/BTG2/pc3 by p-Erk1/2 induces Pin-1 binding in cytoplasm and cell death. J Biol Chem. 2005;280(22):21256-21263.

30. Mongroo PS, et al. IMP-1 displays cross-talk with $\mathrm{K}$-Ras and modulates colon cancer cell survival through the novel proapoptotic protein CYFIP2. Cancer Res. 2011;71(6):2172-2182.

31. Kerley-Hamilton JS, Pike AM, Li N, DiRenzo J, Spinella MJ. A p53-dominant transcriptional response to cisplatin in testicular germ cell tumor-derived human embryonal carcinoma. Oncogene. 2005;24(40):6090-6100.

32. Kawase T, et al. PH domain-only protein PHLDA3 is a p53-regulated repressor of Akt. Cell. 2009;136(3):535-550.

33. Jackson RS 2nd, Cho YJ, Stein S, Liang P. CYFIP2, a direct p53 target, is leptomycin-B sensitive. Cell Cycle. 2007;6(1):95-103.

34. Chau BN, et al. Identification of SULF2 as a novel transcriptional target of $\mathrm{p} 53$ by use of integrated genomic analyses. Cancer Res. 2009;69(4):1368-1374.

35. Sermeus A, Michiels C. Reciprocal influence of the $\mathrm{p} 53$ and the hypoxic pathways. Cell Death Dis. 2011;2:e164.

36. Lion $\mathrm{M}$, et al. Interaction between $\mathrm{p} 53$ and estradiol pathways in transcriptional responses to chemotherapeutics. Cell Cycle. 2013;12(8):1211-1224.

37. Tu Z, et al. Embryonic and hematopoietic stem cells express a novel SH2-containing inositol 5 -phosphatase isoform that partners with the Grb2 adapter protein. Blood. 2001; 98(7):2028-2038.

38. Miller LD, et al. An expression signature for p53 status in human breast cancer predicts mutation status, transcriptional effects, and patient survival. Proc Natl Acad Sci U S A. 2005;102(38):13550-13555.

39. Curtis $\mathrm{C}$, et al. The genomic and transcriptomic architecture of 2,000 breast tumours reveals novel subgroups. Nature. 2012; 486(7403):346-352.

40. Silwal-Pandit L, et al. TP53 mutation spectrum in breast cancer is subtype specific and has distinct prognostic relevance. Clin Cancer Res. 2014;20(13):3569-3580.

41. Liu Q, et al. SHIP is a negative regulator of growth factor receptor-mediated PKB/Akt activation and myeloid cell survival. Genes Dev. 1999;13(7):786-791.

42. Brooks R, et al. SHIP1 inhibition increases immunoregulatory capacity and triggers apoptosis of hematopoietic cancer cells. J Immunol. 2010;184(7):3582-3589.

43. Chen EY, Mazure NM, Cooper JA, Giaccia AJ. Hypoxia activates a platelet-derived growth factor receptor/phosphatidylinositol 3-kinase/Akt pathway that results in glycogen synthase kinase-3 inactivation. Cancer Res. 2001;61(6):2429-2433.

44. Stegeman $\mathrm{H}$, et al. Activation of AKT by hypoxia: a potential target for hypoxic tumors of the head and neck. BMC Cancer. 2012;12(1):463.

45. Yao W, et al. Association of $\mathrm{p} 53$ expression with prognosis in patients with esophageal squamous cell carcinoma. Int J Clin Exp Pathol. 2014;7(10):7158-7163.

46. Ohki R, et al. PHLDA3 is a novel tumor suppressor of pancreatic neuroendocrine tumors. Proc Natl Acad Sci U S A. 2014;111(23):E2404-E2413.

47. Cheung EC, Ludwig RL, Vousden KH. Mitochondrial localization of TIGAR under hypoxia stimulates HK2 and lowers ROS and cell death. Proc Natl Acad Sci U S A. 2012; 109(50):20491-20496.

48. Viernes DR, Choi LB, Kerr WG, Chisholm JD. Discovery and development of small molecule SHIP phosphatase modulators. Med Res Rev. 2014;34(4):795-824.

49. Wennemers M, et al. Hypoxia regulation of phosphokinases and the prognostic value of pAKT in breast cancer. Int J Biol Markers. 2013;28(2):151-160.

50. Stiles BL. PI-3-K and AKT: Onto the mitochondria. Adv Drug Deliv Rev. 2009;61(14):1276-1282.

51. Pal SK, Reckamp K, Yu H, Figlin RA. Akt inhibitors in clinical development for the treatment of cancer. Expert Opin Investig Drugs. 2010;19(11):1355-1366.

52. Fruman DA, Rommel C. PI3K and cancer: lessons, challenges and opportunities. Nat Rev Drug Discov. 2014;13(2):140-156.

53. Diaz R, et al. The novel Akt inhibitor Palomid 529 (P529) enhances the effect of radiotherapy in prostate cancer. $\mathrm{Br} \mathrm{JCancer}$. 2009;100(6):932-940

54. Chen W, Wu S, Zhang G, Wang W, Shi Y. Effect of AKT inhibition on epithelial-mesenchymal transition and ZEB1-potentiated radiotherapy in nasopharyngeal carcinoma. Oncol Lett. 2013;6(5):1234-1240

55. Zhang T, et al. Inhibition of PI3 kinases 


\section{RESEARCH ARTICLE}

enhances the sensitivity of non-small cell lung cancer cells to ionizing radiation. Oncol Rep. 2010;24(6):1683-1689.

56. Prevo R, et al. Class I PI 3 kinase inhibition by the pyridinylfuranopyrimidine inhibitor PI-103 enhances tumor radiosensitivity. Cancer Res. 2008;68(14):5915-5923.

57. Hamilton G, et al. AKT regulates NPM dependent ARF localization p53mut stability in tumors.
Oncotarget. 2014;5(15):6142-6167.

58. Fokas E, et al. Dual inhibition of the PI3K/mTOR pathway increases tumor radiosensitivity by normalizing tumor vasculature. Cancer Res. 2012;72(1):239-248.

59. Bencokova Z, Kaufmann MR, Pires IM, Lecane PS, Giaccia AJ, Hammond EM. ATM activation and signaling under hypoxic conditions. Mol Cell Biol. 2009;29(2):526-537.
The Journal of Clinical Investigation

60. Benjamini Y, Hochberg Y. Controlling the false discovery rate - a practical and powerful approach to multiple testing. J Roy Stat Soc B Met. 1995;57(1):289-300.

61. Pastorek J, et al. Cloning and characterization of $\mathrm{MN}$, a human tumor-associated protein with a domain homologous to carbonic anhydrase and a putative helix-loop-helix DNA binding segment. Oncogene. 1994;9(10):2877-2888. 